\title{
Stagnant-lid convection with diffusion and dislocation creep rheology: Influence of a non-evolving grain size
}

\author{
Falko Schulz, ${ }^{1,2}$ Nicola Tosi, ${ }^{1,2}$ Ana-Catalina Plesa ${ }^{2}$ and Doris Breuer ${ }^{2}$ \\ ${ }^{1}$ Department of Astronomy and Astrophysics, Technische Universität, Berlin, Germany \\ ${ }^{2}$ Institute for Planetary Research, German Aerospace Center (DLR), Berlin, Germany.E-mail: falko.schulz@dlr.de
}

Accepted 2019 September 16. Received 2019 September 12; in original form 2019 January 23

\begin{abstract}
SUMMAR Y
Heat transfer in one-plate planets is governed by mantle convection beneath the stagnant lid. Newtonian diffusion creep and non-Newtonian dislocation creep are the main mechanisms controlling large-scale mantle deformation. Diffusion creep strongly depends on the grain size $(d)$, which in turn controls the relative importance of the two mechanisms. However, dislocation creep is usually neglected in numerical models of convection in planetary mantles. These mostly assume linear diffusion creep rheologies, often based on reduced activation parameters (compared to experimental values) that are thought to mimic the effects of dislocation creep and, as a side benefit, also ease the convergence of linear solvers. Assuming Mars-like parameters, we investigated the influence of a non-evolving grain size on Rayleigh-Bénard convection in the stagnant lid regime. In contrast to previous studies based on the Frank-Kamentskii approximation, we used Arrhenius laws for diffusion and dislocation creep - including temperature as well as pressure dependence - based on experimental measurements of olivine deformation. For $d \lesssim 2.5 \mathrm{~mm}$, convection is dominated by diffusion creep. We observed an approximately equal partitioning between the two mechanisms for $d \approx 5 \mathrm{~mm}$, while dislocation creep dominates for $d \gtrsim 8 \mathrm{~mm}$. Independent estimates of an average grain size of few $\mathrm{mm}$ up to $1 \mathrm{~cm}$ or more for present-day Mars suggest thus that dislocation creep plays an important role and possibly dominates the deformation. Mimicking dislocation creep convection using an effective linear rheology with reduced activation parameters, as often done in simulations of convection and thermal evolution of Mars, has significant limitations. Although it is possible to mimic mean temperature, mean lid thickness and Nusselt number, there are important differences in the flow pattern, root mean square velocity, and lid shape. The latter in particular affects the amount and distribution of partial melt, suggesting that care should be taken upon predicting the evolution of crust production when using simplified rheologies. The heat transport efficiency expressed in terms of the Nusselt number as a function of the Rayleigh number is thought to depend on the deformation mechanisms at play. We show that the relative volume in which dislocation creep dominates has nearly no influence on the Nusselt-Rayleigh scaling relation when a mixed rheology is used. In contrast, the flow pattern influences the Nusselt number more strongly. We derived a scaling law for the Nusselt number based on the mean lid thickness $(\langle L\rangle)$ and on the effective Rayleigh number $\left(\mathrm{Ra}_{\text {eff }}\right)$ obtained by suitably averaging the viscosity beneath the stagnant lid. We found that the Nusselt number follows the scaling $\mathrm{Nu}=0.37\langle L\rangle^{-0.666} \mathrm{Ra}_{\mathrm{eff}}^{0.071}$ regardless of the deformation mechanism.
\end{abstract}

Key words: Heat flow; Numerical modelling; Planetary interiors; Rheology: mantle.

\section{INTRODUCTION}

The rheology of planetary mantles is a function of composition, temperature, pressure, strain rate and grain size, whose relative importance strongly depends on the underlying deformation mechanism. The two main deformation mechanisms that are thought to control the large-scale dynamics of the mantle of terrestrial bodies are diffusion and dislocation creep (e.g. Karato \& Wu 1993; Hirth \& Kohlstedt 2003; Karato \& Jung 2003). The former dominates for small grain sizes and at low stresses; the latter dominates in the presence of large grains and high stresses. The dynamics of the mantle is locally controlled by the mechanism that delivers the 
highest deformation and hence the lowest viscosity. Diffusion creep causes a random distribution of grain orientations, which leads to effectively isotropic media. On the contrary, dislocation creep promotes grain alignment and anisotropy (e.g. Blackman \& Kendall 2002; Kaminski et al. 2004). The observation of seismic anisotropy in the Earth's upper mantle and the lack thereof in the bulk of the lower mantle are usually interpreted as an indication that the deformation is controlled by dislocation creep in the former and by diffusion creep in the latter (e.g., Karato et al. 1995; Karato 1998; McNamara et al. 2001). The fact that the pressure and temperature range covered by the mantles of Mercury, the Moon and Mars lies well within that of the Earth's upper mantle has led some authors to hypothesize that deformation in the mantle of these bodies - all of which operate in the stagnant lid mode of convection-could be largely controlled by dislocation rather than diffusion creep (e.g. Reese et al. 1999; Redmond \& King 2007; Zhang \& O'Neill 2016).

Regional-scale models of the dynamics of the Earth's interior routinely use complex rheologies that account for the effects of multiple deformation mechanisms, including diffusion and dislocation creep (e.g. Karato et al. 2001; Billen \& Hirth 2007; Č́žková et al. 2007; Tosi et al. 2015). However, despite few exceptions (e.g. Alisic et al. 2010; Foley \& Bercovici 2014; Dannberg et al. 2017), the majority of global-scale models of thermal convection in the Earth and terrestrial planets are based on the use of a diffusion creep rheology with constant grain size (e.g., Laneuville et al. 2013; Tosi et al. 2013; Zhang et al. 2013; Nakagawa \& Tackley 2015; Plesa et al. 2015; Zhang \& O'Neill 2016; Scheinberg et al. 2018). Understanding under which conditions mantle convection operates via diffusion or dislocation creep along with the role of these rheologies in controlling heat transfer is thus crucial to reliably simulate the thermal evolution of terrestrial bodies.

The effects of purely non-linear, power-law rheologies have been investigated by various authors. Christensen (1984) compared thermal convection models with Newtonian and non-Newtonian rheology and found that the global characteristics of the latter could be reproduced by a Newtonian rheology with properly adjusted temperature and pressure dependence (see Section 2.4). Reese et al. (1998) and Solomatov \& Moresi (2000) showed that the heat transport efficiency is affected more strongly by the internal Rayleigh number when a non-Newtonian rheology is used. Thermal convection calculations in a mobile-lid regime with a weakly temperature-dependent viscosity combining Newtonian and non-Newtonian creep have been performed by van den Berg et al. (1993, 1995). They showed that the rheology becomes more and more dominated by nonNewtonian creep upon increasing the vigour of convection (i.e. the Rayleigh number) and that lateral variations of the viscosity tend to be controlled by the strain-rate dependence rather than by temperature. However, the increase in convection vigour was achieved simply by increasing the characteristic temperature contrast across the domain, without accounting for a detailed analysis of the grain size, which allows for a more direct exploration of the relative importance of diffusion and dislocation creep.

Furthermore, most of the above studies used flow laws based on the so-called Frank-Kamenetskii approximation, a linearized form of the Arrhenius law that facilitates the convergence of numerical algorithms in the presence of large viscosity variations. Yet it is well known that this approximation tends to smooth the strong viscosity gradients associated with the more realistic Arrhenius law (e.g. Noack \& Breuer 2013; Stein \& Hansen 2013).

The grain size plays a crucial role in determining whether the deformation is governed by linear diffusion or non-linear dislocation creep. Mineral grains evolve according to thermomechanical conditions, undergoing normal growth and reduction due to dynamic recrystallization (e.g. Karato 2012). Austin \& Evans (2007), Ricard \& Bercovici (2009) and Rozel et al. (2011) proposed a theoretical framework to determine the evolution of the grain size in a self-consistent way based on local conditions of temperature, pressure and strain rate. Notably, the dependence of the grain size on the strain-rate makes diffusion creep also non-Newtonian. Rozel (2012) applied the model of Rozel et al. (2011) in the context of numerical simulations of thermal convection with a composite diffusion-dislocation creep rheology. By computing an equilibrium grain size based on the local stress field - without accounting for the actual time-evolution of the grain size-Rozel (2012) investigated the impact of such a composite and grain size-dependent rheology on inducing different convection regimes, from mobile lid to episodic and stagnant lid. In particular, he found that the additional non-linearity induced by the grain size can lead to episodes of surface mobilization without the need to invoke mechanisms of plastic yielding. Dannberg et al. (2017) performed simulations of convection in the present-day Earth's mantle, including a model of grain size evolution. They compared the model outcomes with viscosity profiles inferred from geophysical inversions and with various seismological observables. They found that the use of such a complex rheology has a strong impact on mantle convection through its influence on the viscosity profile, on its lateral variations, and on the shape of up- and downwellings. Foley \& Bercovici (2014) derived scaling laws for Rayleigh-Bénard convection including grain damage. Despite the use of a diffusion creep rheology, convection with grain damage is non-Newtonian since the grain size is calculated in dependence of the deformation work. They showed that the heat flux scales according to a power-law dependence on the Rayleigh number which is larger than typically assumed. The derived scaling law is based on a number of parameters that are incorporated in the models. For example, the damage-to-healing ratio, the internal viscosity, the Frank-Kamentskii parameter, and the sensitivity of the viscosity regarding the grain size. The latter also determines the power-law dependence on the Rayleigh number.

Despite these efforts, grain size evolution models still require the choice of (or the inversion for) a number of poorly known parameters, such as the activation enthalpy of grain growth or the amount of energy dissipated during deformation in relation to the work that can be used for grain size reduction, which crucially affect the behaviour of grains (and hence the dominant deformation mechanism) but are difficult to measure in the laboratory (e.g. Evans et al. 2001).

A comprehensive treatment of stagnant-lid convection based on fixed grain sizes and experimentally derived flow laws for olivine in the diffusion and dislocation creep regime is presently lacking. Numerous studies of convection and thermal evolution of terrestrial planets use an effective diffusion creep rheology with modified activation enthalpy in place of the non-linear dislocation creep rheology that is thought to be relevant for Mercury, the Moon and Mars (e.g. Elkins-Tanton et al. 2005; Šrámek \& Zhong 2010; Roberts \& Barnouin 2012; Zhang et al. 2013; Scheinberg et al. 2014, 2018; Sekhar \& King 2014; Rolf et al. 2016; Zhang \& O’Neill 2016; Citron et al. 2018). The activation energy (when only temperaturedependent viscosity is considered) or activation enthalpy (when both temperature and pressure dependence are taken into account) of dislocation creep are typically reduced by a variable amount-usually a factor of two to three but without explicit justification-based on the work of Christensen (1984). Yet, the extent to which this approximation is applicable, especially for stagnant lid convection, is an open question. Whether or not it can reliably capture the heat 
flux, mantle temperature and characteristics of the flow of an actual non-linear model remains to be investigated.

In the context of Mars, a few attempts have been made to estimate a characteristic grain size of the present-day mantle on the base of dissipation models constrained by the tidal quality factor and Love number $k_{2}$ (Nimmo \& Faul 2013; Khan et al. 2018; Plesa et al. 2018). The inferred present-day grain sizes can vary from few $\mathrm{mm}$ up to $1 \mathrm{~cm}$ or more. Using numerical convection models to determine whether diffusion or dislocation creep controls the deformation over this range of grain sizes can help guiding the choice of the rheology in future modelling efforts.

In addition, various studies have shown that the scaling relation between Nusselt and Rayleigh number depends on the stressexponent. However, these studies are based on purely dislocation creep rheology (e.g. Reese et al. 1998; Solomatov \& Moresi 2000). It is unclear whether or not this is also the case in the presence of a composite rheology, where the relative importance of diffusion and dislocation creep is controlled by varying the grain size.

In this work, we conduct a systematic investigation of the effects of different but non-evolving grain sizes on simple models of Rayleigh-Bénard convection in the stagnant-lid regime. We use various rheologies, from purely diffusion creep to purely dislocation creep through mixed ones accounting for both mechanisms simultaneously. In Section 2, we present the governing equations (2.1) and the rheological formulation (2.2) along with its nondimensionalization (2.3). We present a strategy to mimic nonNewtonian rheology with a Newtonian one with modified activation parameters (2.4), introduce various definitions of the effective Rayleigh number that are used in scaling laws for convective heat transfer (2.5), and provide solidus and liquidus temperatures that we use to estimate the amount of partial melt produced in the different models (2.6). Our numerical setup is presented in Section 3 and the results in Section 4. After a qualitative description of convection simulations in the diffusion and dislocation creep regime and a comparison between the two (4.1-4.3), we show to what extent dislocation creep can be mimicked by a Newtonian rheology (4.4) and present scaling laws for heat transfer based on various NusseltRayleigh relationships (4.5). In Section 5, we summarize our results and discuss their implications for convection in the mantle of Mars.

\section{THEORY AND MODEL}

\subsection{Equations of thermal convection}

We model convection in a planetary mantle by solving the conservation equations of mass, momentum and thermal energy. Under the extended Boussinesq approximation with infinite Prandtl number (e.g. King et al. 2010), denoting non-dimensional variables with the prime symbol, the conservation equations read:

$$
\begin{array}{r}
\nabla \cdot \vec{u}^{\prime}=0 \\
-\nabla p^{\prime}+\nabla \cdot\left(\eta^{\prime}\left(\nabla \vec{u}^{\prime}+\left(\nabla \vec{u}^{\prime}\right)^{\mathrm{T}}\right)\right)+\mathrm{Ra}_{\mathrm{r}} T^{\prime} \hat{\mathrm{e}}_{z}=0 \\
\frac{\partial T^{\prime}}{\partial t^{\prime}}+\vec{u}^{\prime} \cdot \nabla T^{\prime}-\nabla^{2} T^{\prime}+\operatorname{Di}\left(T^{\prime}+T_{0}^{\prime}\right) u_{z}^{\prime}-\frac{\operatorname{Di}}{\operatorname{Ra}_{\mathrm{r}}} \Phi=0
\end{array}
$$

where $T^{\prime}$ is the temperature, $p^{\prime}$ the dynamic pressure, $\vec{u}^{\prime}$ the velocity, $u_{z}^{\prime}$ the vertical component of the velocity, $\hat{e}_{z}$ the vertical unit vector, $\eta^{\prime}$ the viscosity and $\Phi=\underline{\sigma}^{\prime}: \dot{\epsilon}^{\prime}$ the viscous heating, with $\underline{\sigma}^{\prime}$ and $\underline{\epsilon}^{\prime}$ being the stress and strain rate tensors, respectively. The (reference)
Rayleigh number and dissipation number are defined as follows:

$\mathrm{Ra}_{\mathrm{r}}=\frac{g \rho \alpha \Delta T D^{3}}{\eta_{\mathrm{r}} \kappa}$

$\mathrm{Di}=\frac{\alpha g D}{c_{p}}$

where $g$ is the gravitational acceleration, $\alpha$ the thermal expansivity, $\Delta T$ the temperature contrast between core-mantle boundary (CMB) and surface, $D$ the mantle thickness, $\kappa$ the thermal diffusivity, $c_{p}$ the specific heat capacity, $\rho$ the density and $\eta_{\mathrm{r}}$ the reference viscosity. Eqs (1)-(3), are obtained as usual by scaling lengths $z$ with the mantle thickness $D$ :

$z=D z^{\prime}$,

the temperature with the contrast $\Delta T$ between $\mathrm{CMB}$ and surface, that is,

$T=T_{0}+\Delta T T^{\prime}$,

where $T_{0}$ is the surface temperature, the viscosity with a characteristic value $\eta_{\mathrm{r}}$ which will be discussed in Section 2.3:

$\eta=\eta_{\mathrm{r}} \eta^{\prime}$

the pressure with the scale

$p=\frac{\kappa \eta_{\mathrm{r}}}{D^{2}} p^{\prime}$

and the time according to the timescale of thermal diffusion, that is,

$t=\left(\frac{D^{2}}{\kappa}\right) t^{\prime}$

By solving the extendend Boussinesq eqs (1)-(3), our models differ from previous ones that are mostly based on the standard Boussinesq approximation. Due to the lack of consideration of a variable density, the extended Boussinesq approximation is considered thermodynamically not consistent, although for a small planet like Mars, characterized by a low dissipation number $(\mathrm{Di}=0.131)$, this issue is of secondary importance. Nevertheless, we checked in all simulations whether or not, in steady state, the viscous heating balances the adiabatic heating (fourth and fifth term in eq. 3 ). We found a maximum deviation between these two global values of less than one percent. We also found nearly the same difference when comparing the top and bottom Nusselt numbers.

\subsection{Composite rheology}

For mantle rocks, the general relation between strain rate $(\underline{\dot{\epsilon}})$ and differential stress $(\underline{\sigma})$ can be expressed as follows:

$\underline{\dot{\epsilon}_{i}}=A_{i} \underline{\sigma}^{n_{i}} d^{-m_{i}} \exp \left(-\frac{E_{i}+P V_{i}}{R T}\right)$,

where $T$ is the temperature, $P$ the hydrostatic pressure, $R$ the gas constant, and $d$ the grain size (all with their dimensional units). Eq. (11) has general validity for a variety of deformation mechanisms such as diffusion creep, dislocation creep, or grain boundary sliding (Karato \& Wu 1993; Hirth \& Kohlstedt 2003). A given deformation mechanism $i$ is characterized by its own stress exponent $n_{i}$, grain size exponent $m_{i}$, activation energy $E_{i}$, activation volume $V_{i}$, and pre-factor $A_{i}$ (see Table 1). The dependence on additional factors such as water fugacity or melt fraction can be lumped together in the pre-factor $A_{i}$. As long as all deformation processes are 
Table 1. Rheological parameters for diffusion and dislocation creep of dry olivine after Hirth \& Kohlstedt (2003) and constant parameters used in all simulations.

\begin{tabular}{lcc}
\hline Parameter & Symbol & Value \\
\hline Activation energy (diffusion) & $E_{1}$ & $375 \mathrm{~kJ} \mathrm{~mol}^{-1}$ \\
Activation volume (diffusion) & $V_{1}$ & $8.2 \times 10^{-6} \mathrm{~m}^{3} \mathrm{~mol}^{-1}$ \\
Grain size exponent (diffusion) & $m_{1}$ & 3.0 \\
Stress exponent (diffusion) & $n_{1}$ & 1.0 \\
Prefactor (diffusion) & $A_{1}$ & $1.5 \times 10^{-15} \mathrm{~m}^{m_{1}}(\mathrm{~Pa} \mathrm{~s})^{-1}$ \\
Activation energy (dislocation) & $E_{2}$ & $530 \mathrm{~kJ} \mathrm{~mol}^{-1}$ \\
Activation volume (dislocation) & $V_{2}$ & $17 \times 10^{-6} \mathrm{~m}^{3} \mathrm{~mol}^{-1}$ \\
Grain size exponent (dislocation) & $m_{2}$ & 0 \\
Stress exponent (dislocation) & $n_{2}$ & 3.5 \\
Prefactor (dislocation) & $A_{2}$ & $1.1 \times 10^{-16} 1 / \mathrm{Pa}^{n_{2} \mathrm{~S}}$ \\
\hline Mantle thickness & $D$ & $1700 \mathrm{~km}^{-2}$ \\
Gravitational acceleration & $\mathrm{g}$ & $3.7 \mathrm{~m} \mathrm{~s}^{-2}$ \\
Temperature drop across the mantle & $\Delta T$ & $2000 \mathrm{~K}$ \\
Surface temperature & $T_{0}$ & $250 \mathrm{~K}$ \\
Reference density & $\rho$ & $3.5 \times 10^{3} \mathrm{~kg} \mathrm{~m}^{-3}$ \\
Thermal expansivity & $\alpha$ & $2.5 \times 10^{-5} 1 \mathrm{~K}^{-1}$ \\
Thermal diffusivity & $\kappa$ & $1.0 \times 10^{-6} \mathrm{~m}^{2} \mathrm{~s}^{-1}$ \\
Specific heat capacity & $c_{p}$ & $1200 \mathrm{~J}\left(\mathrm{~kg} \mathrm{~K}^{-1}\right.$ \\
\hline Reference temperature & $T_{\mathrm{r}}$ & $2250 \mathrm{~K}$ \\
Reference pressure & $P_{\mathrm{r}}$ & $20.015 \mathrm{GPa}^{-1}$ \\
\hline
\end{tabular}

independent of each other, the total strain rate is given by the sum of the strain rates associated with the various deformation mechanisms, that is,

$\dot{\epsilon}_{\mathrm{tot}}=\sum_{i} \dot{\dot{\epsilon}}_{i}$.

Therefore, the mechanism that delivers the highest strain rate tends to control the deformation.

Assuming isotropy, the (scalar) dynamic viscosity $\eta$ relates strain rate and stress via the constitutive relation $\underline{\sigma}=2 \eta \underline{\dot{\epsilon}}$. Since the stress exponent can be different for each deformation process, we cannot directly determine the effective viscosity from the total strain rate (12). We thus obtain a viscosity law for each deformation mechanism separately (iso-strain model):

$\eta_{i}=\frac{1}{2 A_{i}^{\frac{1}{n_{i}}}} \dot{\epsilon}^{\frac{1-n_{i}}{n_{i}}} d^{\frac{m_{i}}{n_{i}}} \exp \left(\frac{E_{i}+P V_{i}}{n_{i} R T}\right)$,

where $\dot{\epsilon}$ is the second invariant of the (total) strain rate tensor. We then calculate the effective viscosity $\eta_{\text {eff }}$ as the harmonic average of the viscosities $\eta_{i}$ associated with the various mechanisms:

$\eta_{\mathrm{eff}}=\left(\sum_{i} \eta_{i}^{-1}\right)^{-1}$

\subsection{Non-dimensionalization of the viscosity}

The viscosity (14) needs to be cast in non-dimensional form in order to be used in eq. (2). We present two different formulations of the non-dimensional viscosity. One is used for parameter studies on activation energy and activation volume (Section 4.4). The other is used when the grain size is investigated (Section 4.1, 4.2 and 4.3).

To non-dimensionalize eq. (13), we scale the length, temperature and time as in Section 2.1 and replace the hydrostatic pressure in eq. (13) with $P=\rho g z$, where $z$ is the depth. The activation energy and volume are thus scaled as follows:

$E=R \Delta T E^{\prime}$,
$V=\frac{R \Delta T}{\rho g D} V^{\prime}$

Introducing the reference viscosity $\eta_{\mathrm{r}}$, the non-dimensional form of eq. (13) reads:

$\eta_{i}^{\prime}=\frac{1}{2 \eta_{\mathrm{r}} A_{i}^{\frac{1}{n_{i}}}}\left(\frac{\kappa}{D^{2}}\right)^{\frac{1-n_{i}}{n_{i}}} d^{\frac{m_{i}}{n_{i}}} \dot{\epsilon}^{\prime \frac{1-n_{i}}{n_{i}}} \exp \left(\frac{E_{i}^{\prime}+z^{\prime} V_{i}^{\prime}}{n_{i}\left(T^{\prime}+T_{0}^{\prime}\right)}\right)$.

Since the grain size only enters the rheological law and does not appear explicitly in the conservation equations, we keep it in dimensional units. Furthermore, it would not be appropriate to use the mantle thickness for scaling the grain size, which typically ranges from millimeters to centimetres.

Note that $\eta_{\mathrm{r}}$ represents just a viscosity that is thought to be characteristic for the system under consideration and that we use to determine the (reference) Rayleigh number $\mathrm{Ra}_{\mathrm{r}}$ (eq. 4). This reference viscosity can also be calculated self-consistently for a given set of reference values of temperature, pressure (or depth), strain rate and grain size:

$\eta_{\mathrm{r}}=\frac{1}{2 A_{i}^{\frac{1}{n_{i}}}}\left(\frac{\kappa}{D^{2}}\right)^{\frac{1-n_{i}}{n_{i}}} d_{\mathrm{r}}^{\frac{m_{i}}{n_{i}}} \dot{\epsilon}_{\mathrm{r}}^{\prime \frac{1-n_{i}}{n_{i}}} \exp \left(\frac{E_{i}^{\prime}+z_{\mathrm{r}}^{\prime} V_{i}^{\prime}}{n_{i}\left(T_{\mathrm{r}}^{\prime}+T_{0}^{\prime}\right)}\right)$.

However, this reference viscosity corresponds exclusively to a specific deformation mechanism $i$. While this is not suited to determine the effective viscosity in the presence of a composite rheology, it is advantageous in studies where only a single deformation mechanism is considered. In such a case, the non-dimensional viscosity reads:

$\eta_{i}^{\prime}=\left(\frac{d}{d_{\mathrm{r}}}\right)^{\frac{m_{i}}{n_{i}}}\left(\frac{\dot{\epsilon}}{\dot{\epsilon}_{\mathrm{r}}}\right)^{\frac{1-n_{i}}{n_{i}}} \exp \left(\frac{E_{i}^{\prime}+z^{\prime} V_{i}^{\prime}}{T^{\prime}+T_{0}^{\prime}}-\frac{E_{i}^{\prime}+z_{\mathrm{r}}^{\prime} V_{i}^{\prime}}{T_{\mathrm{r}}^{\prime}+T_{0}^{\prime}}\right)$.

If the grain size is constant in time, the first term on the righthand side of eq. (19) can be eliminated. We will use this formula only when a Newtonian rheology is investigated. In such a case, we write:

$\eta_{i}^{\prime}=\exp \left(\frac{E_{i}^{\prime}+z^{\prime} V_{i}^{\prime}}{T^{\prime}+T_{0}^{\prime}}-\frac{E_{i}^{\prime}+z_{\mathrm{r}}^{\prime} V_{i}^{\prime}}{T_{\mathrm{r}}^{\prime}+T_{0}^{\prime}}\right)$.

eq. (20) is often used in mantle convection models with viscosity based on diffusion creep (e.g. Tosi et al. 2013; Plesa et al. 2015). The advantage is that the pre-factor $A_{i}$ (including water fugacity and melt fraction among other material parameters) and the grain size $d$ no longer appear in the equation and can be simply controlled through the Rayleigh number. This simplifies parametric studies based on variations of the activation energy and activation volume because no co-variation to the pre-factor $A_{i}$ is necessary. In fact, if $A_{i}$ were kept in the equation, it would have to be adjusted to ensure that the viscosity at reference conditions remains the same upon varying the activation parameters.

Upon treating composite rheologies or rheologies with explicit grain size dependence, we will calculate the effective nondimensional viscosity as the harmonic average of the viscosities (17) associated with each of the deformation mechanisms considered, that is,

$\eta_{\mathrm{eff}}^{\prime}=\left(\sum_{i} B_{i} d^{-\frac{m_{i}}{n_{i}}} \dot{\epsilon}^{\prime \frac{n_{i}-1}{n_{i}}} \exp \left(-\frac{E_{i}^{\prime}+z^{\prime} V_{i}^{\prime}}{n_{i}\left(T^{\prime}+T_{0}^{\prime}\right)}\right)\right)^{-1}$

with

$B_{i}=2 \eta_{\mathrm{r}} A_{i}^{\frac{1}{n_{i}}}\left(\frac{\kappa}{D^{2}}\right)^{\frac{n_{i}-1}{n_{i}}}$. 
Using eq. (21) facilitates parametric studies based on the variation of the grain size (see Sections 4.1 and 4.3). After choosing an arbitrary reference viscosity, the reference Rayleigh number (4) can be computed and kept fixed in all simulations, while only the grain size is systematically varied. Therefore such reference Rayleigh number is not necessarily representative of the convecting system that is being considered.

\subsection{Mimicking the viscosity of dislocation creep}

Christensen (1984) found that considering non-linear rheologies dependent on strain rate causes the viscosity to exhibit a weaker dependence on temperature and pressure with respect to a linear rheological model with the same activation parameters. Specifically, he showed that the effects of a dislocation creep rheology characterized by a certain activation energy $E_{\text {dis }}$ and activation volume $V_{\text {dis }}$ (briefly, activation enthalpy $H$ ) can be mimicked by an effective diffusion creep rheology with reduced activation enthalpy

$\eta^{*}=\frac{1}{2 A^{*}} \exp \left(\zeta \frac{E_{\mathrm{dis}}+P V_{\mathrm{dis}}}{R T}\right)$,

where $A^{*}$ is an effective pre-factor and $\zeta$ is the factor by which the activation enthalpy is reduced. Yet Christensen (1984) also concluded that the reduction factor $(\zeta)$ must be carefully chosen. For example, he found $\zeta \approx 1 / 2$ when the viscosity is dominated by pressure and $\zeta \approx 1 / 3$ when it is dominated by temperature. From a computational point of view, using an effective diffusion creep rheology to mimic dislocation creep is particularly convenient. It avoids the need to run complex and time-consuming non-linear models and facilitates the convergence of linear solvers because of the reduced viscosity contrasts due to the low activation enthalpy. Indeed this simplification has been adopted by several authors, particularly in the context of simulations and thermal evolution of Mars' mantle (e.g. Elkins-Tanton et al. 2005; Srámek \& Zhong 2010; Scheinberg et al. 2014; Sekhar \& King 2014; Zhang \& O’Neill 2016; Citron et al. 2018).

The numerical experiments presented by Christensen (1984), however, were based on suitably chosen activation parameters and not on their experimental values, which at present are fairly well known, at least for olivine (e.g. Karato \& Wu 1993; Hirth \& Kohlstedt 2003). Furthermore, the activation parameters used by Christensen (1984) led to mobile lid or sluggish lid convection and it is not clear whether these would need to be scaled in the same way when dealing with a purely stagnant lid regime.

In order to understand whether and to what extent stagnant lid convection in a purely dislocation creep regime can be mimicked using an effective diffusion creep model with modified activation enthalpy, we follow here a similar strategy as Christensen (1984). We base our analysis on experimental values of the activation energy and volume of olivine deforming in the dislocation creep regime according to Hirth \& Kohlstedt (2003). As we will show in Section 4.2, at the strain rates encountered in our simulations, these parameters always lead to the formation of a stagnant lid.

For dislocation creep described by eq. (13) with $n=3.5, m$ $=0$, activation energy $E_{\text {dis }}$ and activation volume $V_{\text {dis }}$, we seek an effective Newtonian rheology (eq. 23) with $n=1$ and $m=0$ (because dislocation creep is independent of the grain size) that is able to reproduce a few key features of the non-Newtonian model. Since both $A^{*}$ and $\zeta$ are unknown, we non-dimensionalize such viscosity law in a standard way as in eq. (20). We varied the reduction factor $\zeta$ between 0.1 and 0.5 . For each value of $\zeta$, we varied the reference Rayleigh number between $3 \times 10^{4}$ and $3 \times 10^{6}$. According to the definition (4) of the Rayleigh number, the pre-factor can be recalculated as

$A^{*}=\frac{\operatorname{Ra}_{\mathrm{r}} \kappa \exp \left(\zeta \frac{E_{\mathrm{dis}}+P_{\mathrm{r}} V_{\mathrm{dis}}}{R T_{\mathrm{r}}}\right)}{2 \alpha \rho g \Delta T D^{3}}$.

We infer best-fitting parameters for the effective diffusion creep model by comparing the mean mantle temperature, Nusselt number, and thickness of the stagnant lid with those derived from the dislocation creep model. The difference between actual dislocation creep and mimicked dislocation creep is then investigated in more detail by comparing the lid shape and flow pattern (see Section 4.4).

Note that for simplicity, in the following sections we will omit the prime symbol when referring to non-dimensional variables unless explicitly stated.

\subsection{Nusselt-Rayleigh scaling}

We quantify the heat transfer efficiency of convection in the presence of different rheologies (pure diffusion creep, pure dislocation creep, or a mixture of the two) by running simulations into steady or statistically steady state and fitting the Nusselt $(\mathrm{Nu})$ and Rayleigh number $(\mathrm{Ra})$ to the classical scaling relationship:

$\mathrm{Nu}=\alpha \mathrm{Ra}^{\beta}$.

With no internal heating and in a Cartesian geometry such as the one we used (Section 3), the Nusselt number measured at a given depth $z_{0}$, coincides with the vertical gradient of the laterally averaged temperature profile $(\langle T\rangle)$, that is,

$\mathrm{Nu}=\left.\frac{\partial\langle T\rangle}{\partial z}\right|_{z_{0}}$,

where $z_{0}$ is typically either one or zero, corresponding to the bottom and top of the domain. Eq. (25) is derived from boundary layer theory (Turcotte \& Oxburgh (1967)). For isoviscous Rayleigh-Bénard convection, the theory predicts $\beta=1 / 3$, while the pre-factor $\alpha$ depends on the aspect ratio of the convective cells (e.g. Roberts 1979).

For convection with variable viscosity, the Rayleigh number is no longer constant for the whole system. In eq. (25) it is thus necessary to adopt a value of $\mathrm{Ra}$ based on a characteristic viscosity of the system. The latter depends on the non-dimensionalization adopted. It must be chosen consistently with the reference temperature and pressure when using eq. (20), or with the pre-factors $B_{i}$ when using eq. (21). Without performing the simulations, however, it is difficult to establish whether the chosen characteristic viscosity is actually representative or not of the system under consideration. For Rayleigh-Bénard convection in a fluid (with variable viscosity) heated from below and cooled from above, this requirement can be fulfilled by choosing as a reference the temperature and pressure at the top or at the bottom of the domain since these are known from the boundary conditions. In a system controlled by dislocation creep or mixed rheologies, the situation is further complicated by the fact that also the strain rates are not known a priori. This renders the choice of the reference strain rate $\dot{\epsilon}_{\mathrm{r}}$ (and hence of the reference viscosity) also somewhat arbitrary, with the inverse of the diffusion time representing a possible option that is still consistent with the characteristics of the system, that is, $\dot{\epsilon}_{\mathrm{r}}=\kappa / D^{2}$.

Here we determine the characteristic viscosity $\eta_{\mathrm{r}}$ a posteriori based on the average viscosity $\langle\eta\rangle$ obtained after reaching steady state. To compute $\langle\eta\rangle$, we test different averaging schemes, namely arithmetic, geometric and harmonic mean, as well as an average 
based on the local strain rate that is used to weight the viscosity. The arithmetic and harmonic mean tend to give more importance to regions of high and low viscosity, respectively, while the geometric mean yields a viscosity that is representative of the nearly isothermal convecting domain. For the last case of viscosity weighted by the strain rate, we follow Parmentier et al. (1976) and compute the average viscosity as

$\langle\eta\rangle_{l}=\frac{\int_{V} \dot{\epsilon}^{l} \eta \mathrm{d} V}{\int_{V} \dot{\epsilon}^{l} \mathrm{~d} V}$,

where $l=2$ and $V$ is the volume of the convecting mantle. With eq. (27), regions of high deformation are weighted more strongly than regions of high viscosity. Eq. (27) has also been used by Christensen (1984), who found $l=1$ also to be a suitable choice, as we well as by Kawada \& Honda (1999).

Independent of the method used, we always exclude the stagnant lid upon averaging the viscosity. To calculate its thickness we use the method proposed by Wong \& Solomatov (2015). This method consists in finding the depth of the highest vertical gradient of the root mean square velocity. Such gradient is then extrapolated to the depth of zero velocity, which is defined as the depth of the stagnant lid.

Based on the averaging method used (arithmetic, geometric, weighted with the strain rate and harmonic), we label the effective Rayleigh number with $\mathrm{Ra}_{\text {ari }}, \mathrm{Ra}_{\mathrm{geo}}, \mathrm{Ra}_{\dot{\epsilon}}, \mathrm{Ra}_{\dot{\epsilon}^{2}}$ and $\mathrm{Ra}_{\text {har }}$. If, on the other hand, it is based on the reference values at the CMB and the reference strain rate $\dot{\epsilon}_{\mathrm{r}}=\kappa / D^{2}$, we write $\mathrm{Ra}_{\mathrm{r}}$.

Since the effective Rayleigh number depends on a suitably averaged viscosity, it can be the same for two models with different activation enthalpy. The temperature and pressure dependence of the viscosity, however, affect the thickness of the stagnant lid. The thicker the lid is, the more the interior is insulated and the smaller is the Nusselt number. Hence, in convection studies with variable viscosity based on the Frank-Kamenetskii approach (e.g. Morris \& Canright 1984; Fowler 1985), a power-law dependence on a rheological parameter $\theta$ (the so-called Frank-Kamenetskii parameter) is introduced in the $\mathrm{Nu}-\mathrm{Ra}$ scaling relation, which takes the following form:

$\mathrm{Nu}=\alpha \theta^{\gamma} \mathrm{Ra}_{\mathrm{i}}^{\beta}$.

The exponent $\gamma$ depends on the shape of the stagnant lid. The studies of Reese et al. $(1998,1999)$ and Solomatov \& Moresi (2000) follow this idea noting additionally that $\beta$ is also a function of the stress exponent $n$, with $\beta$ that increases with $n$. In this way, both the dependence of the viscosity on temperature (through $\theta$ ) and on the stress exponent (through $\beta$ ) are included in the scaling relation.

We can convert our activation energy and volume into the FrankKamenetskii parameter. This can be expressed as (e.g. Reese et al. 1999)

$$
\begin{aligned}
\theta & =\left|\Delta T \frac{\mathrm{d}(\ln \eta)}{\mathrm{d} T}\right| \\
& =\frac{\Delta T\left(E+P_{i} V\right)}{R T_{i}^{2}}-\frac{P_{i} V}{R T_{i}},
\end{aligned}
$$

where $P_{i}$ is the pressure at the base of the top thermal boundary layer and $T_{i}$ the internal mantle temperature.

These values define the minimum viscosity of the system which in turn is typically used to define the internal Rayleigh number $\left(\mathrm{Ra}_{\mathrm{i}}\right)$ in eq. (28). In order to use this relation, we first determine the base of the upper thermal boundary layer. As the temperature is subject to strong fluctuation near the boundary-especially if this has a steep slope-we determine $T_{i}$ and $P_{i}$ in the middle of the convecting cell. Both values can than be used to calculate $\mathrm{Ra}_{\mathrm{i}}$ and $\theta$. Since the laterally averaged temperature and viscosity profiles are strongly influenced by the cold part of the stagnant lid, we emphasize that their use is not effective to determine $T_{i}$ and $P_{i}$.

\subsection{Partial melting}

As we shall see below, different values of the grain size- - hence different rheologies - lead to important modifications of the thickness and shape of the stagnant lid. These in turn can affect the amount and distribution of partial melt generated in the sublithospheric mantle. In view of the potential development of our models to treat the long-term thermal evolution of Mars and other stagnant lid bodies, including the history of crust production (e.g. Laneuville et al. 2013; Plesa \& Breuer 2014; Rolf et al. 2016; Padovan et al. 2017), after each simulation reached statistical steady-state (Section 3), we also computed melt fractions $(\varphi)$ assuming a simple linear increase between solidus $\left(T_{\mathrm{sol}}\right)$ and liquidus $\left(T_{\mathrm{liq}}\right)$, that is,

$\varphi=\frac{T-T_{\text {sol }}}{T_{\text {liq }}-T_{\text {sol }}}$.

To compute $\varphi$, we used the peridotite solidus introduced by Ruedas \& Breuer (2017), which is appropriate for the Martian mantle:

$T_{\text {sol }}=0.118912 P^{3}-6.37695 P^{2}+130.33 P+1340.38$,

and the dry liquidus of Katz et al. (2003):

$T_{\text {liq }}=-2 P^{2}+45 P+2053.15$,

where the temperature is expressed in $\mathrm{K}$ and $P$ is the hydrostatic pressure in GPa.

After computing the melt fraction $\varphi_{i}$ in every cell $i$ of the domain, we compute the total melt volume fraction $\left(V_{\varphi}\right)$ simply as:

$V_{\varphi}=\frac{1}{V} \sum_{i} \varphi_{i} \delta V_{i}$

where $\delta V_{i}$ is the volume of the $i$ th cell and $V$ the total volume of the domain. Since we work with a square 2-D grid (see Section 3), $\delta V_{i}$ should be regarded as areas and $V=1$.

\section{NUMERICAL SETUP}

We used our finite volume code Gaia (Hüttig et al. 2013) to solve the governing eqs (1)-(3) in a 2-D box with aspect-ratio one, free-slip boundary conditions, isothermal top and bottom boundaries, and reflective side-walls. The domain was meshed with a uniform grid of $200 \times 200$ nodes. We ran all simulations assuming Mars-like parameters with a temperature contrast across the domain $(\Delta T)$ of $2000 \mathrm{~K}$ and a mantle thickness $(D)$ of $1700 \mathrm{~km}$. To compute the viscosity, we assumed rheological parameters characteristic of dry olivine according to Hirth \& Kohlstedt (2003). These are reported in Table 1 along with the remaining constants.

When the classical implementation of the non-dimensional viscosity was used (eq. 20), we set the reference temperature $\left(T_{\text {ref }}\right)$ to $2250 \mathrm{~K}$ and the reference pressure $\left(P_{\text {ref }}\right)$ to $22.015 \mathrm{GPa}$, corresponding to the $\mathrm{CMB}$ condition. Otherwise we chose a reference viscosity of $10^{21} \mathrm{~Pa} \mathrm{~s}$ in (22), corresponding to a reference Rayleigh number of $3.18 \times 10^{6}$.

We started all simulations setting a constant internal temperature of $1650 \mathrm{~K}$ supplemented by two thermal boundary layers with a 
thickness of $300 \mathrm{~km}$. A sinusoidal perturbation with an amplitude of 0.03 was superimposed to initiate convection. We tracked all simulations until they reached a steady or statistically steady state.

With the given rheological parameters (Table 1), the viscosity can increase by more than 50 orders of magnitude across the mantle. Since most of the viscosity increase is due to the temperature drop within the stagnant lid, we cut the viscosity when it increases by more then 10 orders of magnitude compared to the lowest viscosity of the system.

\section{RESULTS}

\subsection{Diffusion creep}

We first ran a set of 37 simulations assuming a diffusion creep rheology where we varied the grain size between 1.0 and $10.0 \mathrm{~mm}$. Due to the power-law dependence of the grain size $(m=3)$, this can lead to viscosity variations of up to three orders of magnitude, depending on the temperature profile. We found that no convection takes place for grain sizes larger than $\sim 9.2 \mathrm{~mm}$. For grain sizes smaller than this value, we observed a convection planform characterized by either one or two stable cells with the exception of runs with a grain size between 6.25 and $7.25 \mathrm{~mm}$, which never reached a stable steady state and exhibited an oscillatory behaviour. This is characterized by the fact that a hot plume rises alternately at the left or right cell wall with large effects on global properties such as mean temperature and Nusselt number. Thus, we will omit these models in the following analysis.

Fig. 1 shows the steady-state distribution of temperature, viscosity and second invariant of the strain rate tensor, as well as the shape of the stagnant lid for three representative values of the grain size. Increasing the grain size, the viscosity becomes larger and the lid thickness increases. For $7.5 \mathrm{~mm} \leq d<9.2 \mathrm{~mm}$, the stagnant lid covers a significant part of the domain. As a consequence, we observe two stable convection cells (Figs 1a and d) in line with the idea that at, low Rayleigh numbers, cells with unitary aspect ratio tend to be more stable than elongated ones (e.g., Grigné et al. 2007). For intermediate grain sizes $(2.75 \mathrm{~mm} \leq d \leq 6.0 \mathrm{~mm})$ we observed a single cell (Figs $1 \mathrm{~b}$ and e), while for the smallest grain sizes that we tested $(1.0 \mathrm{~mm} \leq d<2.5 \mathrm{~mm})$ we observed a time-dependent flow characterized by two (unstable) cells (Figs 1c and f).

As expected, the decrease of the lid thickness upon reducing the grain size - which corresponds to increasing the Rayleigh number-leads to a clear increase of the area where the temperature exceeds the solidus. While for the largest grain size of $9 \mathrm{~mm}$, no melt can be generated (Fig. 1a), the partial melt regions become larger for grain sizes of 5 (Fig. 1b) and $2.25 \mathrm{~mm}$ (Fig. 1c).

\subsection{Dislocation creep}

When the grain size is sufficiently large, diffusion creep becomes unimportant and the deformation is controlled by dislocation creep. Therefore, in the presence of a composite rheology (see Section 4.3), the lowest heat flux that can be achieved by varying the grain size corresponds to that of a pure dislocation creep model. This implies that models with a composite rheology, in contrast to the pure diffusion creep models discussed above, cannot become conductive upon varying the grain size unless the pure dislocation creep model does.

Fig. 2 shows snapshots of temperature, viscosity and strain rate of this end-member case. Only one convection cell forms. Although dislocation creep is characterized by larger activation energy and activation volume than diffusion creep, the viscosity field is less sensitive to temperature and pressure variations due to the stress exponent $n$ in the Arrhenius term (eq. 13).

A comparison of the distribution of the viscosity (Fig. 2b) and strain rate (Fig. 2c) clearly shows that the regions where the viscosity is lowest correspond to the highest strain rates attained at the left upwelling plume and in the area of horizontal shearing flow beneath the stagnant lid.

In contrast to the diffusion creep models of Fig. 1, here the stagnant lid is flat and thick enough to completely prevent the temperature from exceeding the solidus.

This model only reaches a statistical steady state. Nusselt number, mean temperature, root mean square velocity, and mean lid thickness are subject to small temporal variations. Averaged over a sufficiently long time span we find $\langle T\rangle=0.814, v_{\text {rms }}=235.1, \mathrm{Nu}$ $=2.51$ and $\langle L\rangle=0.276$. Mean temperature and rms-velocity are computed below the stagnant lid.

\subsection{Composite rheology}

As for the diffusion creep simulations, we varied the grain size between 1.0 and $10.0 \mathrm{~mm}$ also in the framework of simulations with composite rheology. We found that this variation is sufficient for convection to be controlled either completely by diffusion (as $d$ approaches $1 \mathrm{~mm}$ ) or almost completely by dislocation creep (as $d$ approaches $10 \mathrm{~mm}$ ). For grain sizes between 1 and $10 \mathrm{~mm}$, Fig. 3(a) displays the fraction of the convective part of the domain (i.e. beneath the stagnant lid) where deformation is controlled by dislocation creep. Up to $d \approx 2.5 \mathrm{~mm}$ diffusion creep dominates. For $d$ between 4.0 and $6.25 \mathrm{~mm}$, the volume fraction of dislocation creep increases rapidly from 20 to 80 per cent. Above $6.25 \mathrm{~mm}$, the increase in volume fraction of dislocation creep is less rapid. Even for grain sizes larger than $9.25 \mathrm{~mm}$ (when pure diffusion creep models became conductive), a small fraction of the domain where strain rates are low is still controlled by diffusion creep.

Like in the diffusion creep simulations, we observed either one or two convection cells corresponding to filled and empty circles in Fig. 3, respectively. For grain sizes between 3.25 and $8.0 \mathrm{~mm}$ we systematically observed a single cell. Outside of this range, we mostly obtained two convection cells with the exception of two cases, namely $d=1.5$ and $9.75 \mathrm{~mm}$. For grain sizes larger than $8 \mathrm{~mm}$, the simulations never reached a stable steady state.

In Fig. 3(b) we compare the melt volume fraction $V_{\varphi}$ (eq. 33) after reaching steady state for all simulations with diffusion creep (blue circles) and composite rheology (red circles). In both cases, increasing the grain size causes a reduction of the convective vigor due to the increase in viscosity. As a result, the stagnant lid becomes thicker and the melt volume fraction decreases. Apart for $d=1 \mathrm{~mm}$, for all the other values of the grain size, $V_{\varphi}$ is systematically smaller for the composite rheology than for pure diffusion creep. The increase of the lid thickness associated with the increase of the grain size (see Fig. 5c) causes partial melt to be suppressed for $d \gtrsim 6 \mathrm{~mm}$.

Fig. 4 shows the steady-state distribution of the temperature (ac), viscosity (d-f), ratio of dislocation to diffusion creep viscosity ( $f=\eta_{\text {dis }} / \eta_{\text {dif }}$ in panels $\left.g-i\right)$ and strain rate $(j-1)$ for three characteristic values of the grain size. The deformation is controlled by the mechanism that delivers the lowest viscosity. Therefore, dislocation creep prevails for $f<1$ while diffusion creep for $f>1$. Upon increasing the grain size above $2 \mathrm{~mm}$, dislocation creep becomes first 

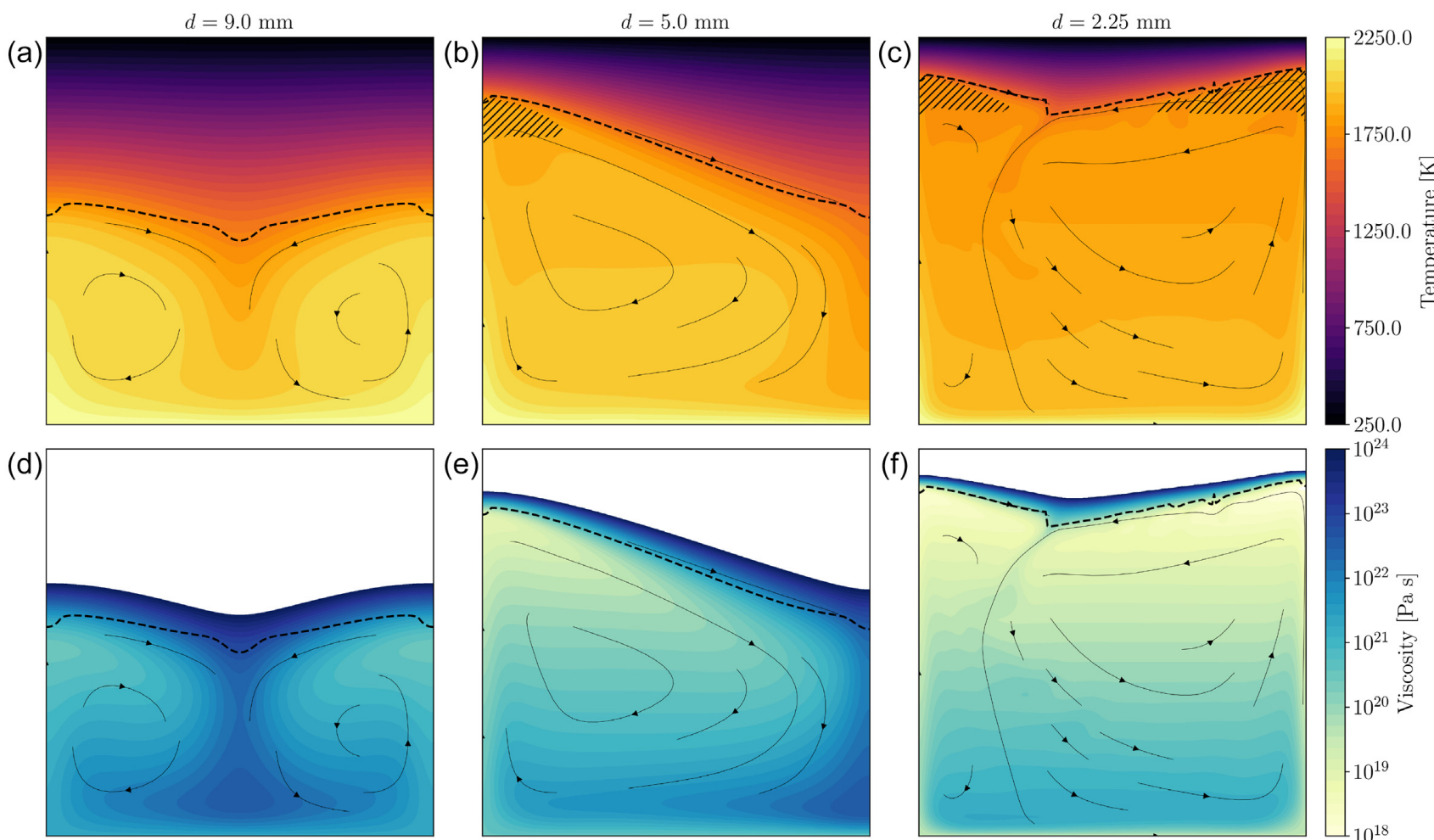

(e)

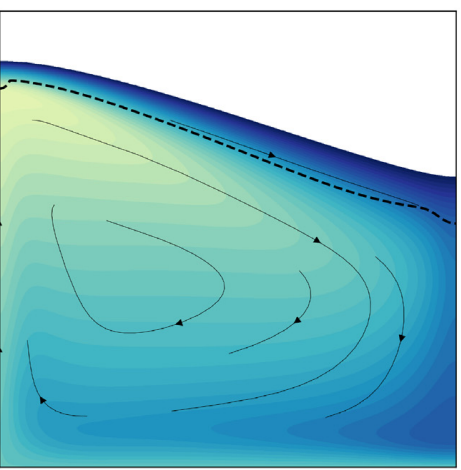

(f)

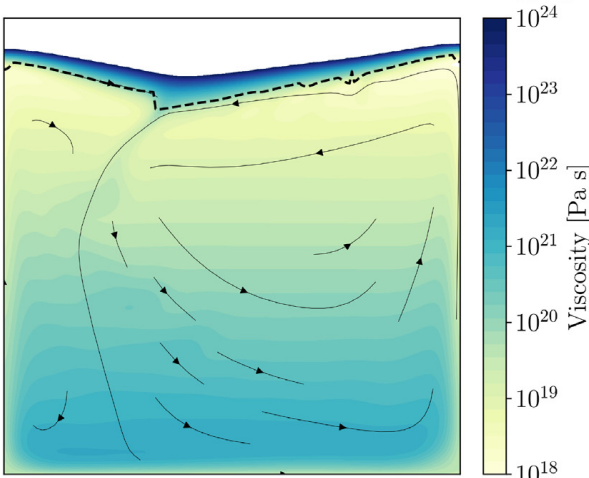

(g)

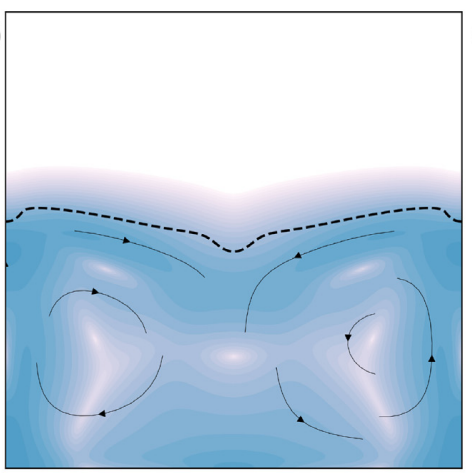

(h)

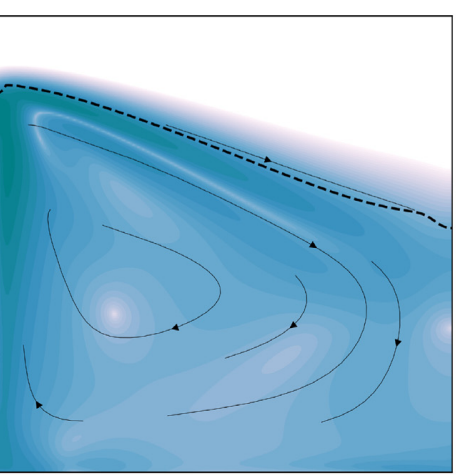

(i)

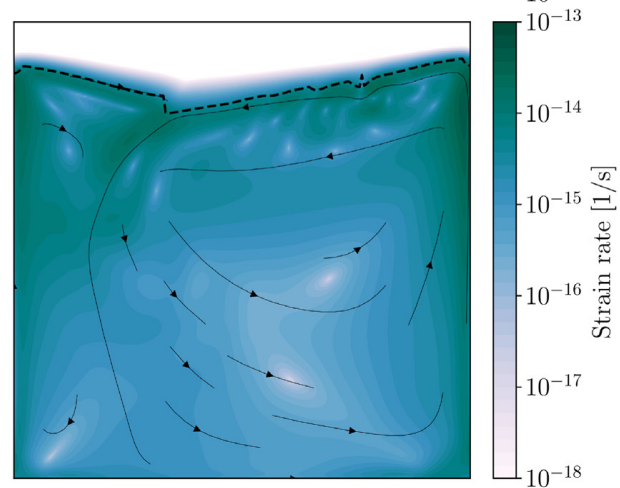

Figure 1. Steady state distribution of temperature $(\mathrm{a}-\mathrm{c})$, viscosity $(\mathrm{d}-\mathrm{f})$ and strain rate $(\mathrm{g}-\mathrm{h})$ for diffusion creep models with different grain sizes. The black dashed line corresponds to the stagnant lid. Hatched areas in panels $(\mathrm{a}-\mathrm{c})$ are regions of partial melt where the solidus is exceeded. Thin black lines represent streamlines. White areas in the viscosity and strain rate plots indicate an upper cut-off of the viscosity at $10^{24} \mathrm{~Pa}$ s, and a lower cut-off of the strain rate at $10^{-18} \mathrm{~s}^{-1}$.

important in a thin region close to the stagnant lid because of relatively high strain rates associated with sublithospheric instabilities, while the bulk of the domain largely deforms via diffusion creep (Fig. 4i). For intermediate values of $d$, the effect of dislocation creep becomes evident in highly strained up- and downwellings, while the bottom and central part of the domain still deform via diffusion creep (Fig. 4h). Only when the grain size exceeds $8 \mathrm{~mm}$ the $\mathrm{CMB}$ region and most of the domain (>90 per cent) are controlled by dislocation creep (Fig. 4g). As anticipated in Fig. 3(b), the increase of $d$ is accompanied by a reduction of the extent of the melt zone (Figs $4 \mathrm{c}$ and $\mathrm{b}$ ), which for $d=9 \mathrm{~mm}$ has completely vanished.

For a given grain size, the composite rheology is systematically characterized by a higher Nusselt number and thinner lid than the diffusion creep rheology (Figs 5a and c). Only when the grain size is set to $1 \mathrm{~mm}$, we observe a slightly higher Nusselt number and thinner lid in the pure diffusion creep case. This discrepancy is explained by the fact that the Nusselt number is affected by the convective planform and number of convection cells. Small differences in the viscosity field of the model with $d=1 \mathrm{~mm}$ cause a slightly different flow pattern. In the diffusion creep case, small convective rolls form below the stagnant lid reducing its thickness, while this is not the case when using a composite rheology. Upon decreasing further the grain size, we thus expect that the models will exhibit the same heat flux.

The mean velocity $v_{\text {rms }}$ (Fig. $5 \mathrm{~d}$ ), determined below the stagnant lid, however, is very similar for grain sizes lower than $\sim 3.5 \mathrm{~mm}$. Otherwise, the composite rheology leads to higher velocities.

The mean temperature, also determined below the stagnant lid, is higher in the diffusion creep case. This can be traced back to the thinner lid of the composite models. A thinner lid provides better heat transfer, which in turn results in a lower temperature of the convecting mantle. Although neither the lid thickness nor the Nusselt number change greatly for grain sizes between 8 and 
(a)

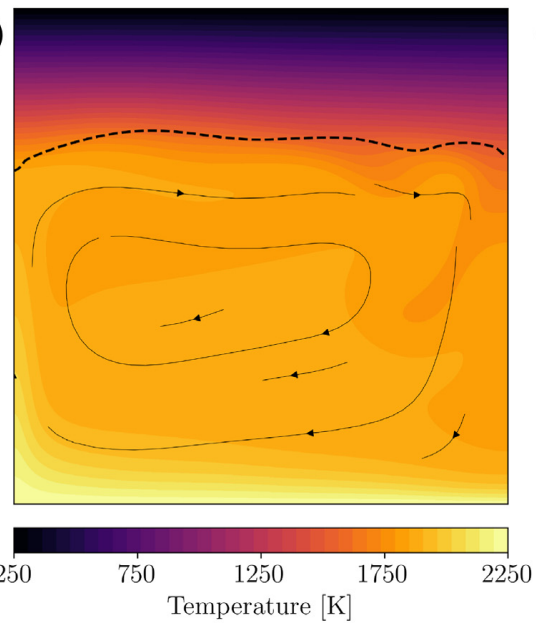

(b)

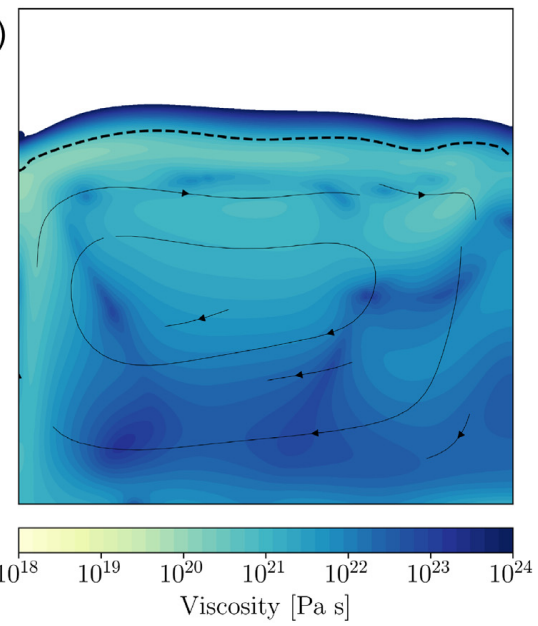

(c)

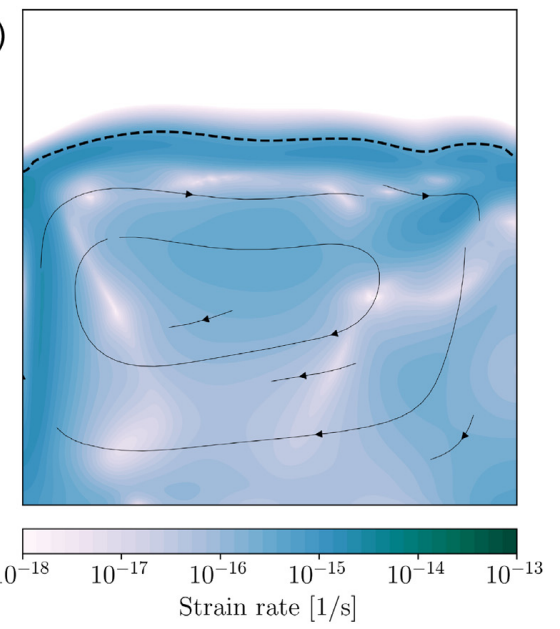

Figure 2. Distribution of temperature (a), viscosity (b) and strain rate (c) for convection with a pure dislocation creep viscosity. The black dashed line corresponds to the stagnant lid and thin black lines represent streamlines. White areas in panel $\mathrm{b}$ and $\mathrm{c}$ indicate an upper cut-off of the viscosity at $10^{24} \mathrm{~Pa} \mathrm{~s}$ and a lower cut-off of the strain rate at $10^{-18} \mathrm{~s}^{-1}$. No partial melt occurs in this model.

(a)
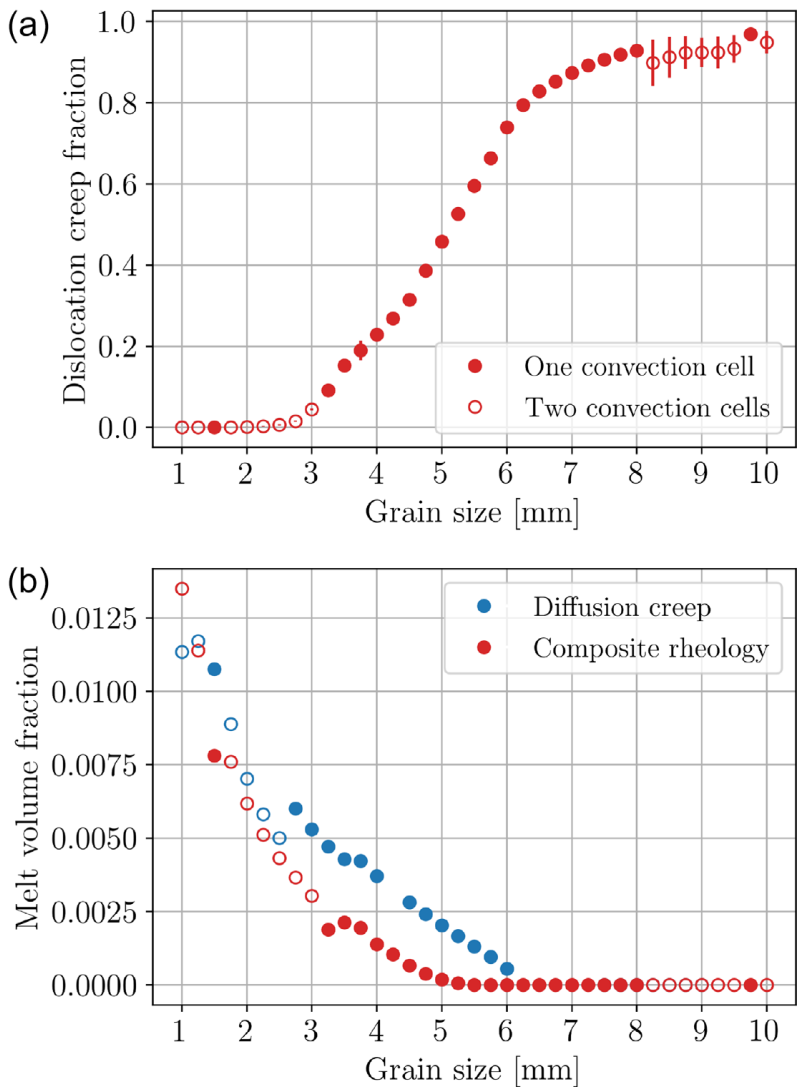

Figure 3. (a) Fraction of the domain where deformation is controlled by dislocation creep for grain sizes between 1 and $10 \mathrm{~mm}$. Filled and empty circles refer to cases characterized by one or two convective cells, respectively. For $d \leq 8 \mathrm{~mm}$, the simulations reached a stable steady state. For larger values, the circles refer to time-averaged values and the error bars to the corresponding variance. (b) Melt volume fraction (eq. 33) as a function of the grain size for the pure diffusion creep runs discussed in Section 4.1 (blue circles) and for the runs with composite rheology of panel a (red circles).

$9 \mathrm{~mm}$ in the composite models, the mean temperature increases dramatically with the change in the number of convection cells. By contrast, it remains nearly constant for $d$ between 4 and $8 \mathrm{~mm}$.
With composite rheology, $\mathrm{Nu}$ and $v_{\text {rms }}$ are systematically larger and the mean lid thickness smaller with resepect to the pure dislocation creep simulation (dashed line in Fig. 5), which is reached asymptotically in the limit of large grains. For grain sizes above $5.5 \mathrm{~mm}$, the diffusion creep models exhibit lower Nusselt numbers than the end-member case of pure dislocation creep. Overall, the more efficient heat transfer observed in the case of composite rheology is mainly due to the thickness and shape of the stagnant lid, which increases more slowly than in the pure diffusion creep models. Finallly, regardless of the rheological model, we observe discontinuous changes with the grain size in the values of all four diagnostic quantities when the flow pattern changes from one to two convection cells and vice versa.

\subsection{Mimicking dislocation creep}

In Fig. 5 we show how the Nusselt number can be controlled by the reference viscosity, which in turn can be changed through the prefactor and hence through the grain size. Based on Fig. 5, by considering only the Nusselt number, we could mimic the heat transfer via dislocation creep with a pure diffusion creep rheology with a grain size of approximately $5.5 \mathrm{~mm}$. However, the temperature and viscosity distributions of this diffusion creep model barely resemble those of dislocation creep (compare Figs 6a and d with Figs $6 \mathrm{~b}$ and e). In contrast to the dislocation creep model where the stagnant lid is nearly flat, the diffusion creep model is characterized by a lid with a steep slope, much thinner over the upwelling than over the downwelling plume. Because of the different temperature structure, while no melt occurs in the dislocation creep case (Fig. 6a), a partial melt region corresponding to the head of the upwelling plume is present in the two models that mimic dislocation creep (Figs 6b and $\mathrm{c}$ ).

As discussed in Section 2.4, in order to find an effective diffusion creep model that better matches the dislocation creep one in terms of global quantities such as Nusselt number, lid thickness, mean temperature and velocity, we followed the approach of Christensen (1984) and ran a series of simulations based on pure diffusion creep using an activation enthalpy corresponding to a fraction $\zeta$ of the activation enthalpy of dislocation creep (eq. 23). A lower activation enthalpy reduces the temperature and pressure dependence of 

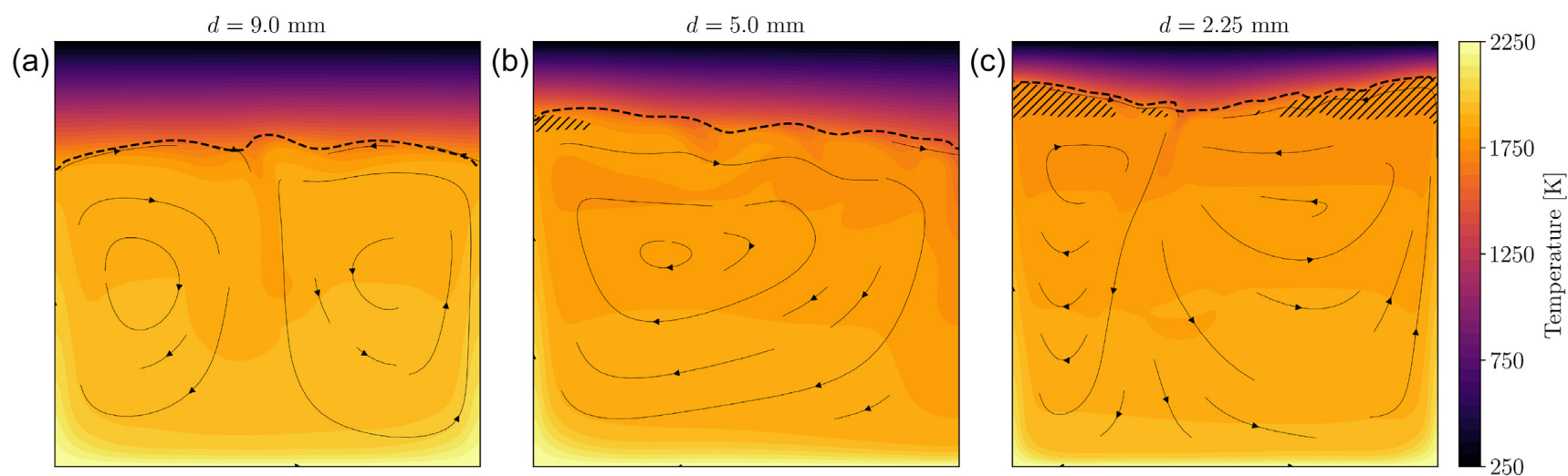

(d)

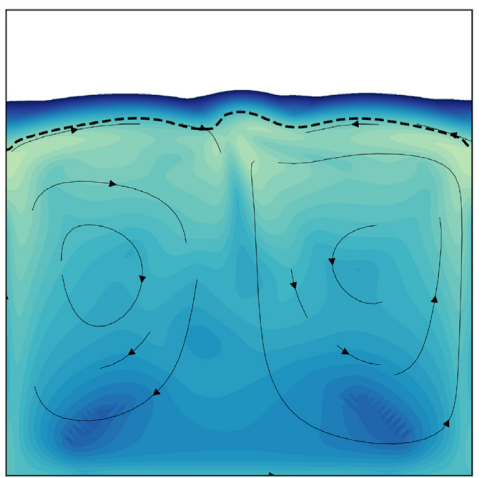

(e)

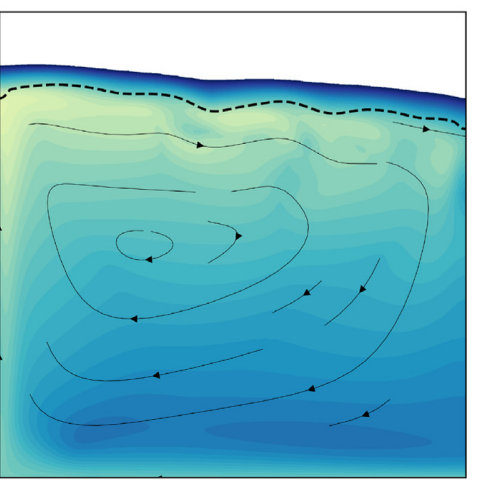

(f)

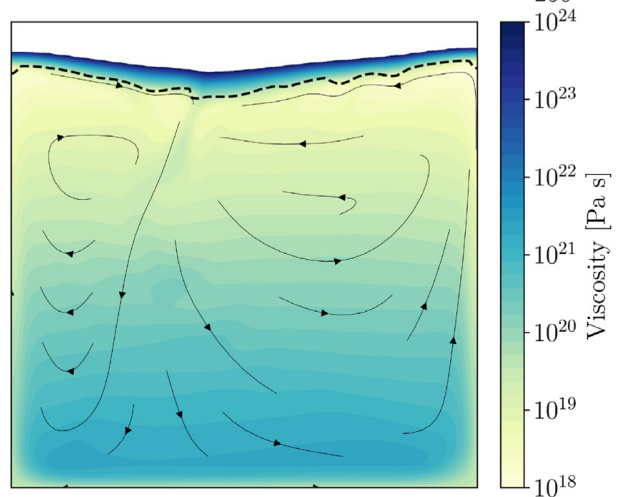

(g)

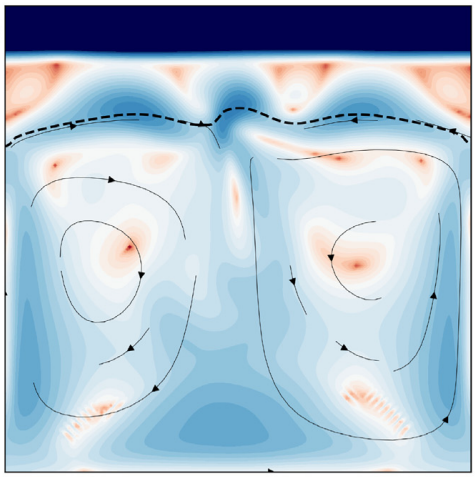

(h)

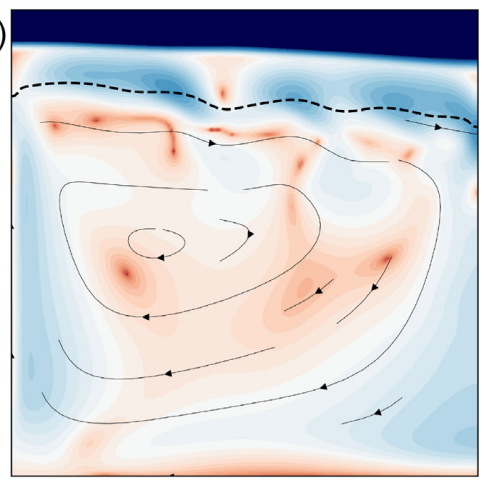

(i)
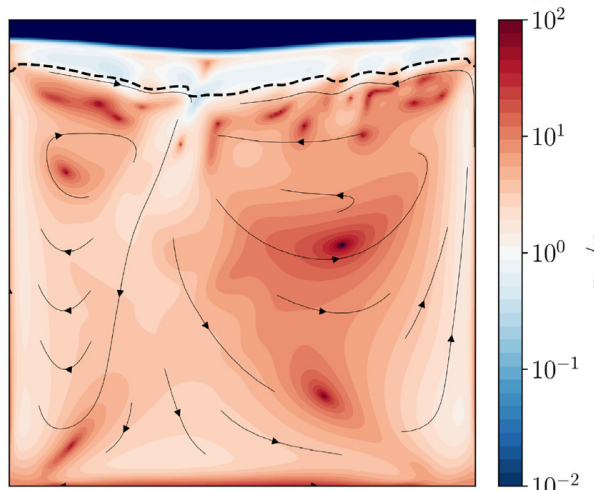

(j)

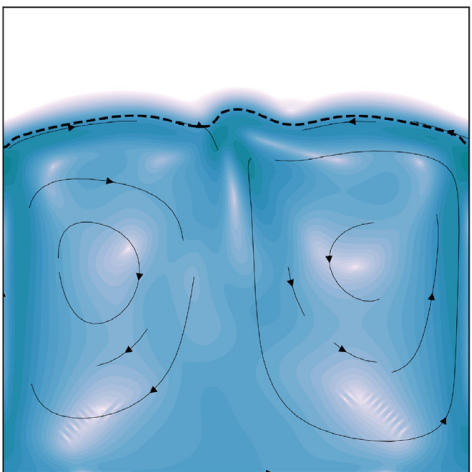

$(\mathrm{k})$

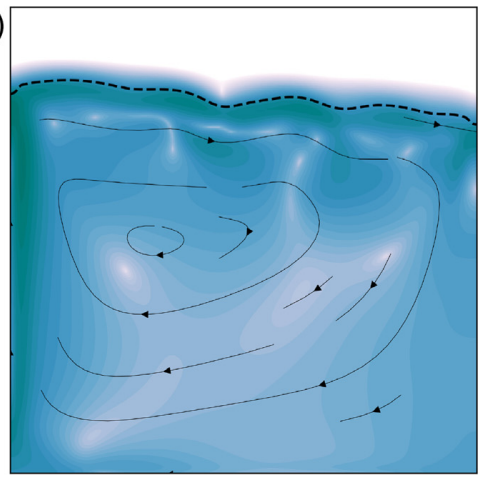

(l)

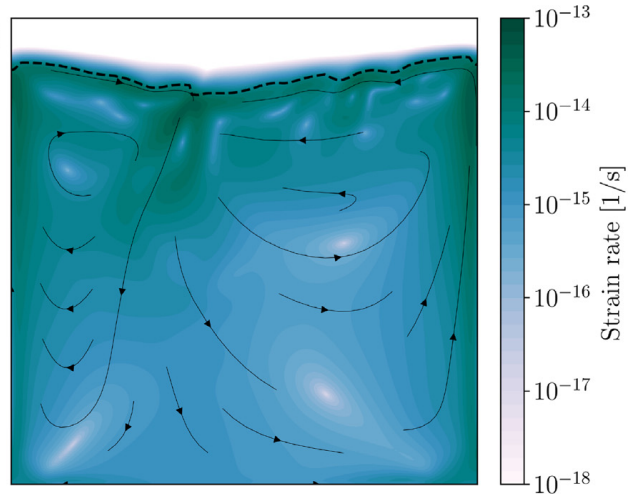

Figure 4. Steady state distribution of temperature (a-c), viscosity $(\mathrm{d}-\mathrm{f})$ and ratio of dislocation creep viscosity $\left(\eta_{\text {dis }}\right)$ to diffusion creep viscosity $\left(\eta_{\text {dif }}\right)$ (g-i) for models with mixed rheology and various grain sizes $d$. The black dashed line corresponds to the stagnant lid and thin black lines represent streamlines. Hatched areas in panels a-c are regions of partial melt where the solidus is exceeded. When the fraction $\eta_{\text {dis }} / \eta_{\text {dif }}$ is smaller one, dislocation creep dominates. Otherwise diffusion creep is the dominant deformation mechanism. 
(a)

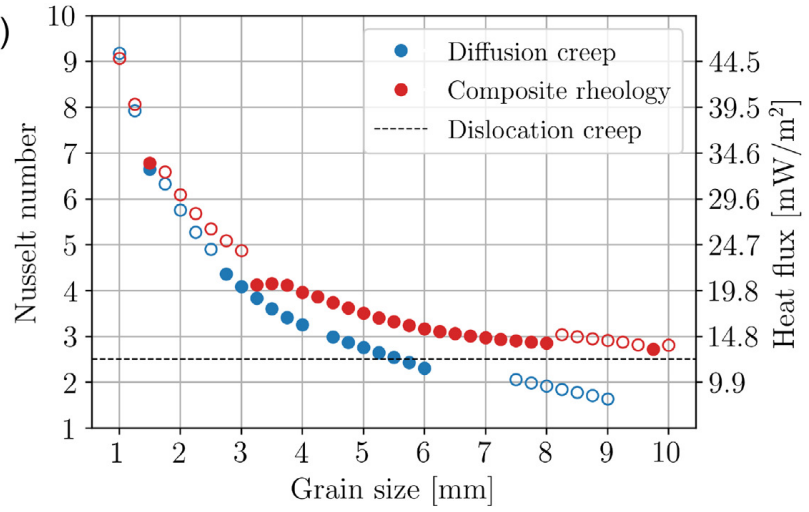

(c)

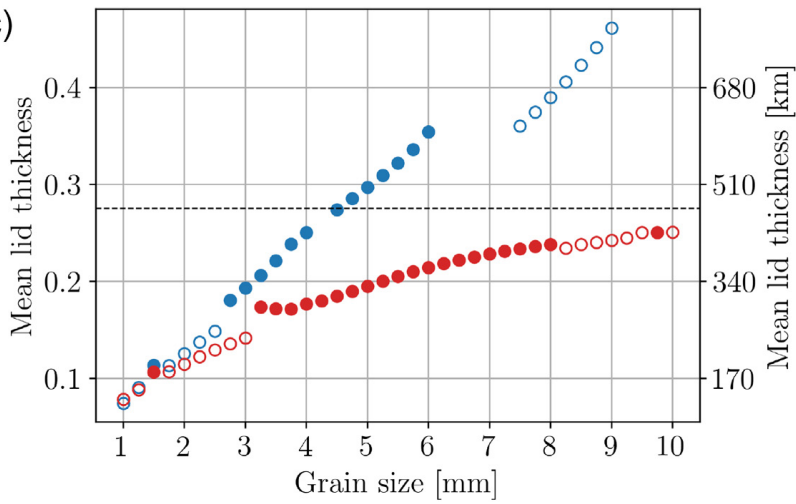

(b)

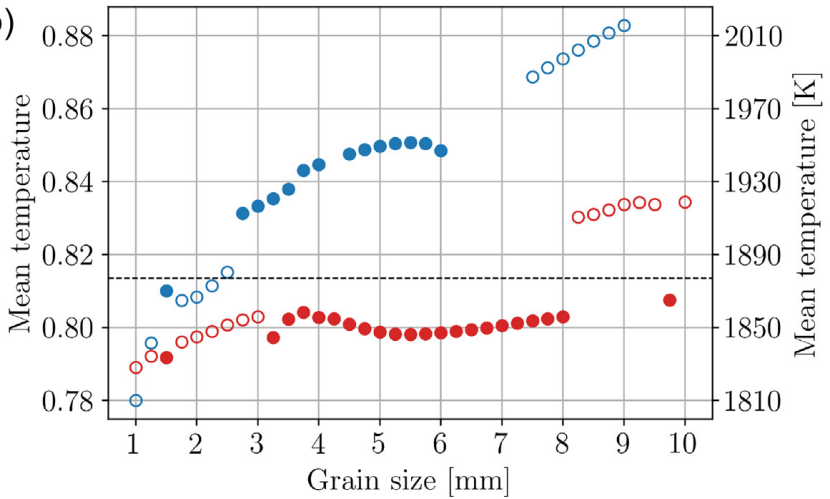

(d)

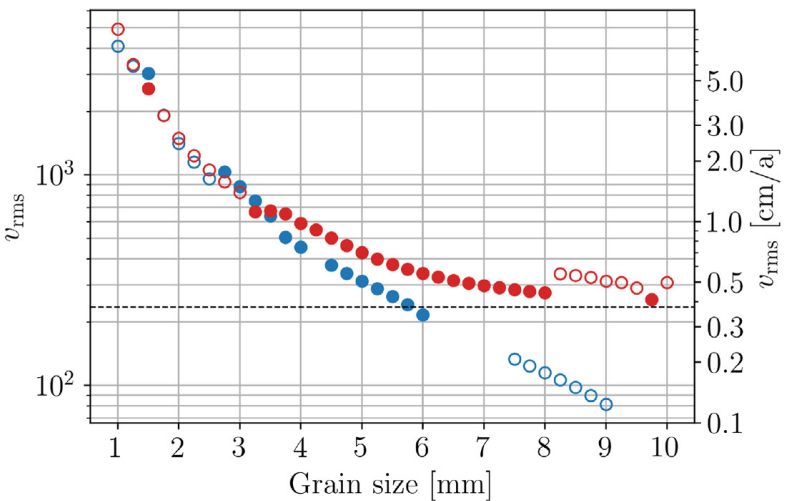

Figure 5. Nusselt number (a), mean temperature (b), mean lid thickness (c) and root mean square velocity (d) as a function of grain size for simulations based on pure diffusion creep (blue circles) and a composite rheology (red circles). Filled and empty circles refer to one or two convection cells in steady state. The horizontal dashed lines refer to the values obtained with the pure dislocation creep model. The root mean square velocity $\left(v_{\text {rms }}\right)$ and the mean temperature are computed below the stagnant lid.

the viscosity and in turn the slope of the stagnant lid. We reduced the activation enthalpy between 90 per cent and 50 per cent, corresponding to $0.1 \leq \zeta \leq 0.5$, and varied simultaneously the reference Rayleigh number between $\mathrm{Ra}_{\mathrm{r}}=3 \times 10^{4}$ and $\mathrm{Ra}_{\mathrm{r}}=3 \times 10^{6}$ for each value of $\zeta$. Fig. 7(a) shows the Nusselt number $(\mathrm{Nu})$ and mean lid thickness $(\langle L\rangle)$ of these effective diffusion creep models over the Nusselt number $\left(\mathrm{Nu}_{\mathrm{dis}}\right)$ and mean lid thickness $\left(\left\langle L_{\mathrm{dis}}\right\rangle\right)$ of the dislocation creep model for different values of $\zeta$ (as indicated by different symbols) and $\mathrm{Ra}_{\mathrm{r}}$ (colour coded). In this representation, the point $(1,1)$ corresponds to a perfect agreement in the lid thickness and Nusselt number. Symbols with a black contour indicate simulations characterized by two convection cells in steady state instead of a single one, which are not suitable to mimic the dislocation creep simulation. Within the blue shaded box, the ratio of the analysed diagnostic quantities obtained with the effective diffusion creep models to the corresponding quantities obtained with the dislocation creep models is below 10 per cent. The best fit to the Nusselt number and mean lid thickness is obtained using $\zeta=0.4$ and $\mathrm{Ra}_{\mathrm{r}} \approx 1 \times 10^{6}$. However, using the above parameters, the agreement with the temperature and viscosity distributions is still not optimal (compare Figs 6a and d with Figs 6c and f). Furthermore, as in the diffusion creep model with $d=5.5 \mathrm{~mm}$, the stagnant lid still exhibits a relatively steep slope. In the one convection cell regime, this slope can only be reduced either by increasing the reference Rayleigh number or decreasing the reduction factor of the activation enthalpy $\zeta$. In both cases the Nusselt number becomes larger and the mean lid thickness smaller compared to the dislocation model (see Fig. 7a). Therefore we conclude that we can mimic dislocation creep in terms of Nusselt number and mean lid thickness, but without accurately reproducing the flow pattern.

In Fig. 7(b) we show the accuracy of the mean temperature and root mean square velocity $\left(v_{\mathrm{rms}}\right)$ of the various models compared to the dislocation creep model. Both values refer to the region below the stagnant lid. Besides the Nusselt number and mean lid thickness, the best fitting models introduced above are within 5 per cent accuracy in mean temperature but largely fail to reproduce $v_{\text {rms }}$ of dislocation creep (compare the positions of the squares in Figs 7a and b).

In synthesis, using an effective diffusion creep model with reduced activation enthalpy to mimic convection in the dislocation creep regime is only possible to some extent. The mimicking procedure yields a good match in terms of mean temperature, mean lid thickness and Nusselt number. However, neither the shape of the lid - and in turn the convective planform - nor the root mean square velocity can be reproduced at the same time. The former has a significant influence on the distribution of partial melt in the sublithospheric mantle (Figs 6a-c), while the latter would likely affect mantle mixing (e.g. Samuel et al. 2011; Samuel \& Tosi 2012).

\subsection{Nusselt-Rayleigh scaling}

\subsubsection{Diffusion creep}

In Fig. 8 we show a $\log$-log diagram of the Nusselt number over the effective Rayleigh number for the diffusion creep models (Section 4.1). The effective Rayleigh number was calculated with the different definitions of the characteristic viscosity introduced in 

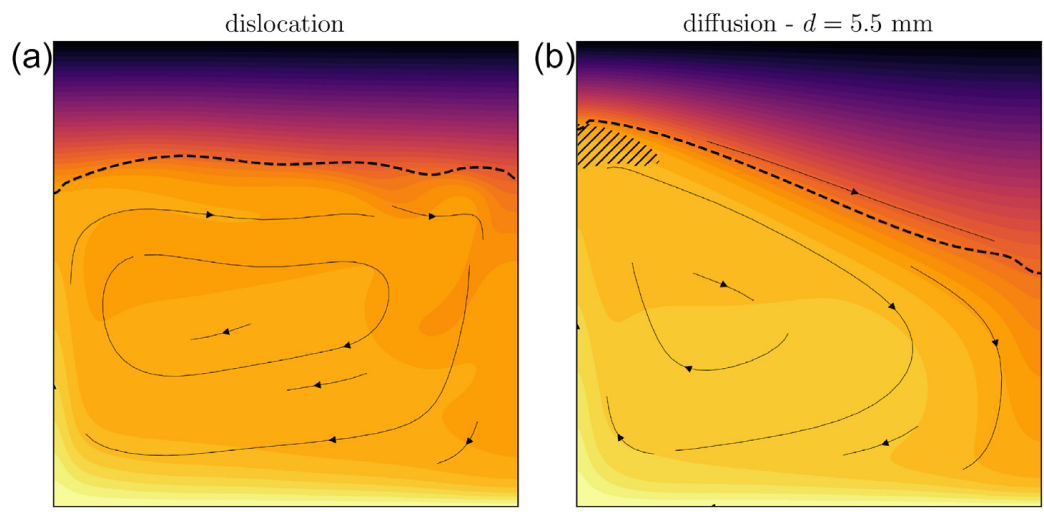

(d)

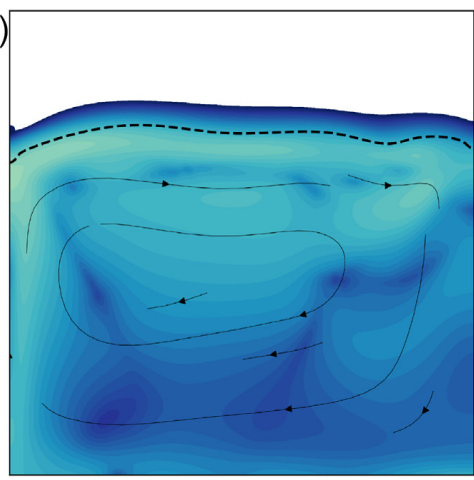

(e)

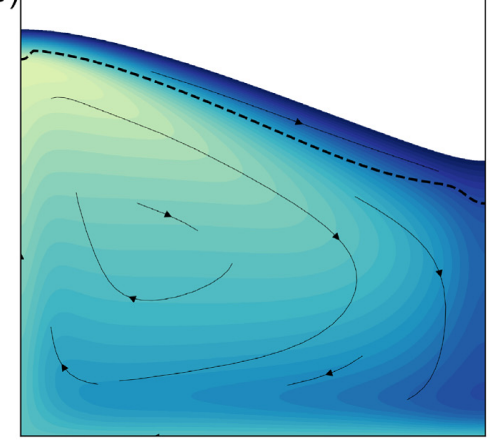

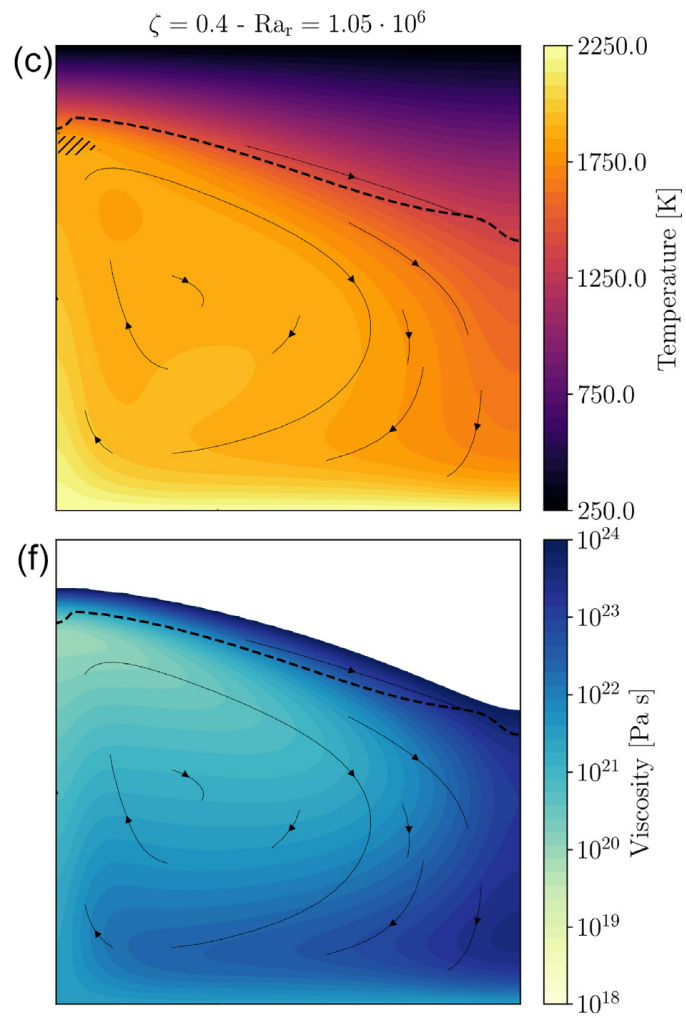

Figure 6. Steady state distribution of temperature and viscosity for the pure dislocation creep model as in panels 2(a, d) and for two diffusion creep models reproducing some of the features of dislocation creep convection (b, e and c, f). The black dashed line corresponds to the stagnant lid, hatched areas mark the melt zone and grey lines represent streamlines.

Section 2.5. As discussed, we fitted the obtained set of Nusselt and Rayleigh numbers to the relation $\mathrm{Nu}=\alpha \mathrm{Ra}^{\beta}$. Fitting parameters are listed in Table 2.

We observe a strong correlation between the change in the slope $(\beta)$ and the change in the number of convection cells. However, the same number of convection cells does not necessarily correspond to the same slope. We thus subdivide the Nusselt-Rayleigh scaling into three regimes (colour-coded in Fig. 8). Regime 1 for low Rayleigh numbers and two convection cells, regime 2 for models with one convection cell and regime 3 when the Rayleigh number is large and two convection cells form. This behaviour is similar to the one we observed in Fig. 5. The simulation using a grain size of $1 \mathrm{~mm}$ (grey circles in Fig. 5), corresponding to the largest Rayleigh number, is not part of regime 3 but most likely marks the beginning of a fourth regime. We thus excluded this value from the scaling relation. The three series of snapshots shown in Fig. 1 reflect these three regimes, with regime 1 obtained with a grain size $d=9 \mathrm{~mm}$, regime 2 with $d=5 \mathrm{~mm}$ and regime 3 with $d=2.25 \mathrm{~mm}$. We also note a slight change in the slope within regime 2 for some choice of the average viscosity (Figs 8a-c). However, we will neglect this change due to the uncertainty of the transition and the low number of simulations within this regime. The fitted curves match well our measured Nusselt number. The coefficient of determination $\left(R^{2}\right)$ of the logarithmic fitting function is generally higher than 99 per cent. The only exception is for regime 2 and the arithmetic mean for which $\mathrm{R}^{2}$ is only 94 per cent.

The slope is in general steepest (i.e. $\beta$ is higher) for small Rayleigh numbers. In the case of $\mathrm{Ra}_{\mathrm{har}}$ and $\mathrm{Ra}_{\mathrm{r}}$, the value decreases with each change of the characteristic flow pattern. When the arithmetic mean is used, the relation between Nusselt and Rayleigh number is not bijective, which suggests that the Rayleigh number alone is not sufficient to determine the heat flux. As already mentioned in Section 2.5, the arithmetic mean tends to give values closer to the maximum viscosity. Therefore, the reduction in $\mathrm{Ra}_{\text {ari }}$ can be explained by the decrease of the internal temperature when the flow pattern changes from two to one convection cell (see Fig. $5 b$ for the change from large to small grains).

The issue with this scaling is that it is only valid for the specific parameters given in Table 1. The diffusion creep runs presented in Section 4.4 with $\zeta=0.1$, for example, yield $\alpha=0.203$ and $\beta=$ 0.218 in the presence of one convection cell (regime 2) when the harmonic average is used to compute the effective Rayleigh number. These values are different from those obtained for regime 2 in the pure diffusion creep case, namely $\alpha=0.041$ and $\beta=0.251$. The reason for this has been explained in Section 2.5 and is due to the different sensitivities to temperature and pressure. The results of Section 4.1 and Section 4.4 can be reconciled by fitting our data to the scaling relation $\mathrm{Nu}=\alpha \theta^{\gamma} \mathrm{Ra}_{\mathrm{i}}^{\beta}$ (eq. 28). The Frank-Kamenetskii parameter, in the steep slope and one-convection-cell regime, varies only slightly between $\theta=24.2$ and $\theta=27.6$ for the diffusion creep simulations (Section 4.1) and between $\theta=6.89$ and $\theta=11.07$ for the models with reduced activation enthalpy (Section 4.4). We found:

$\mathrm{Nu}=0.278 \theta^{-0.4} \mathrm{Ra}_{\mathrm{i}}^{0.203}$.

The maximal deviation of the calculated Nusselt numbers compared to the measured ones is 6.7 per cent where the standard deviation is 0.093. Using the Rayleigh number based on the harmonic average $\left(R a_{h a r}\right)$ instead of $R a_{i}$, we obtain:

$\mathrm{Nu}=0.219 \theta^{-0.581} \mathrm{Ra}_{\text {har }}^{0.262}$, 

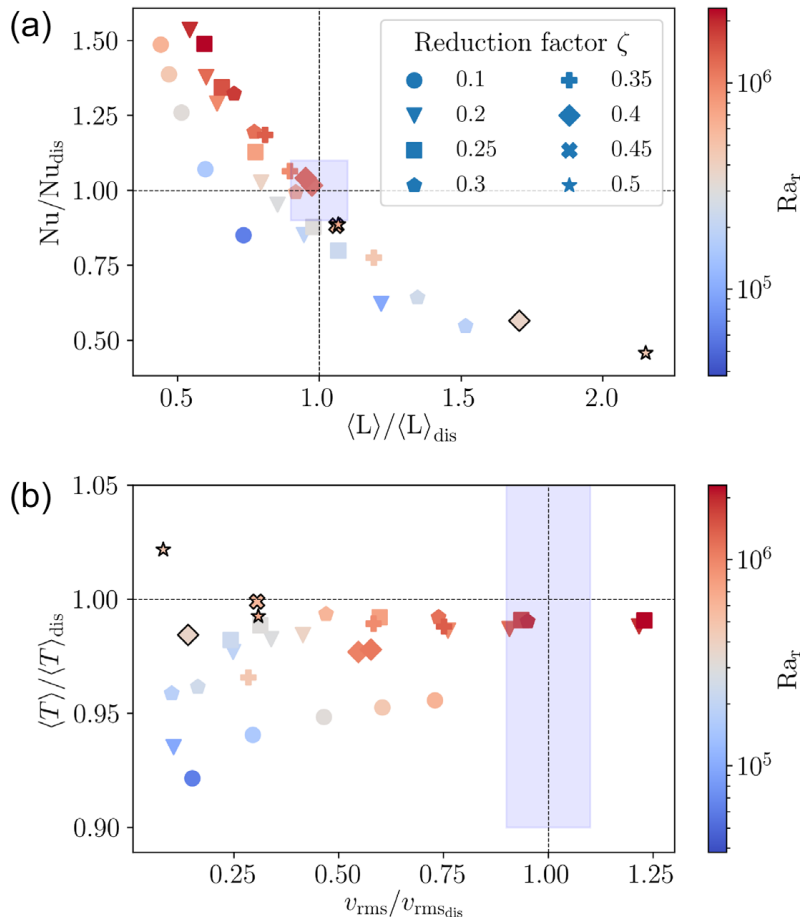

Figure 7. (a) Nusselt number $(\mathrm{Nu})$ and mean stagnant lid thickness $(\langle L\rangle)$ for effective diffusion creep models over Nusselt number and lid thickness for the dislocation creep model $\left(\mathrm{Nu}_{\mathrm{dis}}\right.$, and $\left\langle L_{\mathrm{dis}}\right\rangle$, respectively). Symbols refer to different fractions $\zeta$ of the activation enthalpy of dislocation creep (eq. 23). Symbols with black contour denote simulations characterized by two convective cells. The blue shaded area corresponds to the region where the analyzed ratios are below 10 per cent. The colour scale indicates the reference Rayleigh number $\left(\mathrm{Ra}_{\mathrm{r}}\right)$. (b) As in panel a but for the mean mantle temperature and root mean square velocity. Both values are determined below the stagnant lid.

with a maximal deviation of 4.2 per cent and a standard deviation of 0.06. In agreement with the studies of Fowler (1985) and Reese et al. (1998), the scaling worsens if no consideration is given to the shape of the stagnant lid, which varies here according to the number of convection cells. In addition both the above studies predicted $\beta$ to be $1 / 5$. This matches well with our result using the internal Rayleigh number $\left(\mathrm{Ra}_{\mathrm{i}}\right)$ and has already been confirmed by numerical studies (e.g. Moresi \& Solomatov 1995). Both theory and numerical models have so far predicted a value for $\gamma$ of -1 , while we found a considerably smaller $\gamma=-0.4$, which is likely due to the fact that, in contrast to previous studies, we also took into account the pressure dependence of the viscosity.

The scaling relations (34)-(35) well reproduce the Nusselt number of the dislocation simulation. For this, the effective Rayleigh is $\mathrm{Ra}_{\text {har, dis }}=2.89 \times 10^{6}$ and the generalized Frank-Kamenetskii parameter is $\theta_{\text {dis }}=12.73$. Using the above values for example into eq. (35) yields $\mathrm{Nu}_{\text {dis, predicted }}=2.499$, which compares well with the value of 2.507 obtained from the numerical dislocation creep simulation. However, since the scaling parameters are predicted as functions of the lid shape (flat versus steep) and dislocation creep is different in this respect from the simulations used to derive eqs (34)(35), care should be taken upon using these relations for dislocation creep.

\subsubsection{Composite rheology}

Fig. 9 shows the Nusselt number over the effective Rayleigh number for simulations using a composite rheology (Section 4.3). We use the temperature and pressure at the CMB as well as the specific grain size and the strain rate $\dot{\epsilon}_{\mathrm{r}}=\kappa / D^{2}$ to define the reference Rayleigh number $\mathrm{Ra}_{\mathrm{r}}$. For such a small strain rate the reference Rayleigh number is comparable to the one of pure diffusion creep (see Fig. 8f). The fitting parameters are listed in Table 3. The black marker in the figure indicates values of the pure dislocation creep model (Section 4.2). This marker is not visible in the plot based on $\mathrm{Ra}_{\mathrm{r}}$ as the corresponding reference Rayleigh number of $\approx 10^{4}$ is out of the displayed range. As in the case of the diffusion creep models, we find a change in the exponent $\beta$ and in the factor $\alpha$ of the $\mathrm{Nu}-\mathrm{Ra}$ relationship when the number of convection cells changes from regime 1 , characterized by two convection cells and low Rayleigh number, to regime 2, characterized by one convection cell and regime 3, where the effective Rayleigh number is large and two convection cells are formed. The characteristics of these three regimes have been shown in Fig. 4 (regime 1: $d=9.0 \mathrm{~mm}$, regime 2: $d=5.0 \mathrm{~mm}$ and regime $3: d=2.25 \mathrm{~mm})$. We calculate again the coefficient of determination $\left(\mathrm{R}^{2}\right)$, which is reported in Table 3. Only the scaling based on the harmonic average provides a very good fit for all three regimes. In regime 1, all other averages suggest that the exponential fit to the Rayleigh number is not as good. Compared to the diffusion creep models, $\alpha$ and $\beta$ are largely similar for regime 2 and 3. The exponent $\beta$ changes only in the third digit, whereas $\alpha$ changes in the second. For these two regimes, the heat flux as a function of the Rayleigh number can be described by a single scaling. The flow pattern does not matter here. As a results of the largely constant scaling parameters in regimes 2 and 3 , and the fact that the dislocation creep fraction decreases from 90 to 0 per cent (Fig. 3, from 8 to $1 \mathrm{~mm}$ ) within these regimes, we conclude that whether or not convection is dominated by diffusion or dislocation creep is irrelevant for the scaling law.

Although the influence of the dislocation creep fraction is small, there are significant differences with respect to the pure diffusion creep case (compare Figs 8 and 9 or Tables 2 and 3, respectively). The differences in $\beta$ are likely due to the sensitivity of the lid thickness to the Rayleigh number rather than to the specific rheology chosen.

As in the last section, we observe that the relationship between Nusselt and Rayleigh number is not bijective. The only exception is when the Rayleigh number is defined at the $\mathrm{CMB}$ using the reference strain rate $\dot{\epsilon}_{\mathrm{r}}$. (Fig. 9f).

We calculated the Frank-Kamentskii parameter based on the activation parameters of dislocation creep. This is a reasonable assumption for regimes 1 and 2 since the upper thermal boundary layer is dominated by dislocation creep. We found $\theta$ to be in the range of 11.4-12.8. However, this range is too small and hardly sufficient to derive an accurate scaling of the Nusselt number as a function of Ra and $\theta$.

\subsubsection{Extending the $\mathrm{Nu}-\mathrm{Ra}$ scaling relation}

The heat transfer efficiency of convection is routinely parametrized according to the Rayleigh number. We have shown that this has limitations. On the one hand, the choice of the simplest relation $\mathrm{Nu}=$ $\alpha \mathrm{Ra}^{\beta}$ can provide a good fit to the simulations, but the exponent $\beta$ is a function of the specific rheology: $\beta$ is lower when the activation enthalpy is small and it also varies for different flow patterns. On the other hand, we find no clear relation between $\alpha$ and $\beta$ and the 

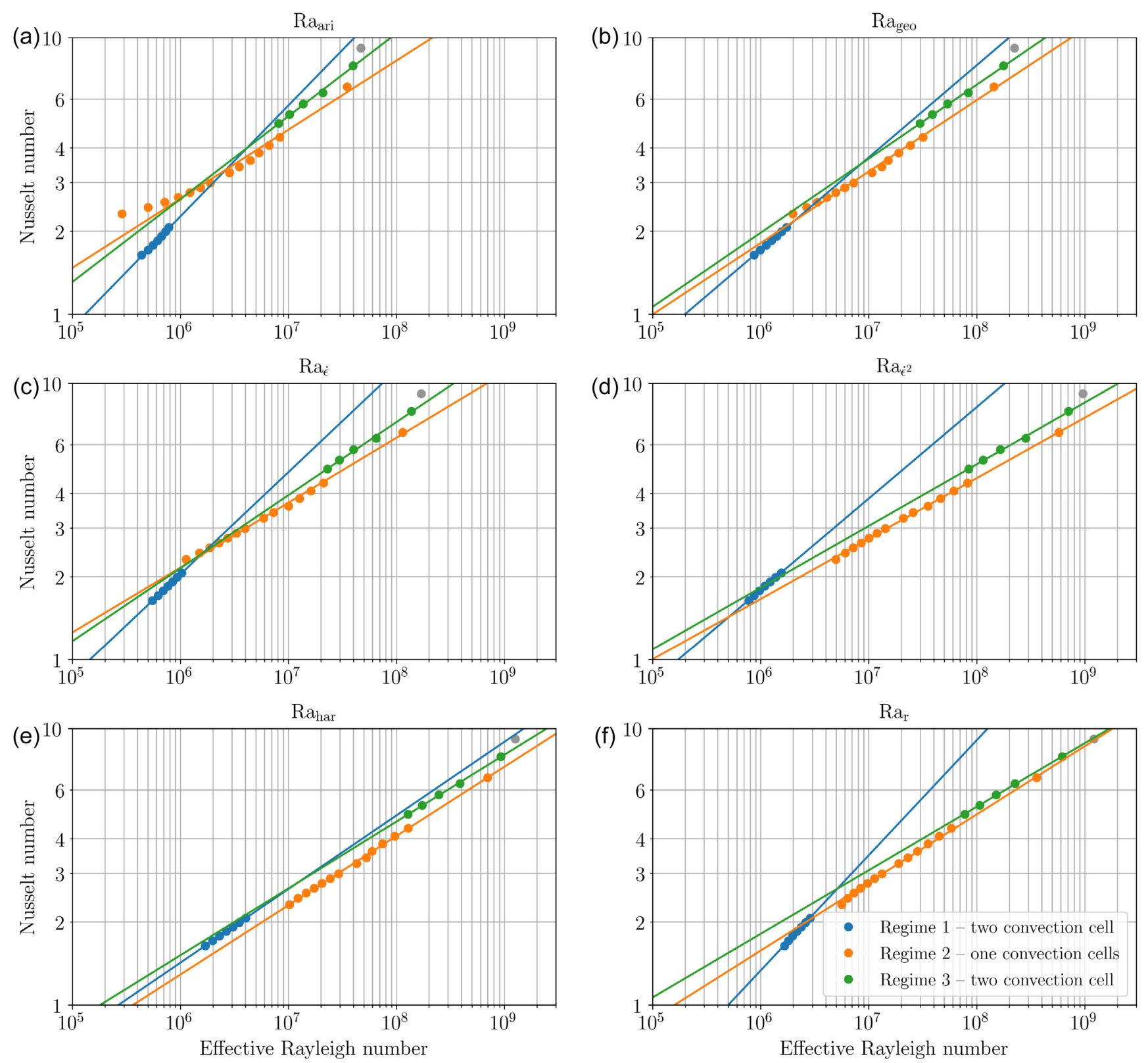

Figure 8. Nusselt number over effective Rayleigh number for pure diffusion creep simulations. In each panel the effective Rayleigh number is calculated by averaging the viscosity (beneath the stagnant lid) in a different way as explained in Section 2.5. In panel (f), no averaging of the viscosity is used and the effective Rayleigh number is simply set equal to the reference Rayleigh number at the bottom of the domain. The three lines are fits to the Nu-Ra scaling relationship (25) corresponding to the three regimes discussed in the text. The grey circle corresponds to $d=1 \mathrm{~mm}$ and belongs to none of the three regimes.

dislocation creep fraction. Including the Frank-Kamenetskii parameter into the scaling relation makes the exponent $\beta$ less dependent on the activation enthalpy, but leaves open the question of how to determine the parameter $\theta$. The latter is a function of the internal temperature $T_{\mathrm{i}}$ and pressure $P_{i}$ (eq. 29), which can be expressed as the depth of the base of the thermal boundary layer $z_{\mathrm{i}}$. Fig. 10 shows how $T_{\mathrm{i}}$ and $z_{\mathrm{i}}$ depend on $\theta$ in the regime of one convection cell with a lid with steep slope for diffusion creep simulations (red crosses) and diffusion creep simulations with reduced activation enthalpy (blue symbols).

The relation of $T_{\mathrm{i}}$ and $z_{\mathrm{i}}$ with $\theta$ is not easily predictable. In particular, for the cases with reduced activation enthalpies, only few simulations are available. Furthermore, $T_{\mathrm{i}}$ and $z_{\mathrm{i}}$ exhibit a very weak dependence on $\theta$ : for small activation enthalpies, $\theta$ remains almost constant upon varying the effective Rayleigh number. The larger is the activation enthalpy, the more is $\theta$ affected by the Rayleigh number.

Instead of eq. (25) or eq. (28), which depend on a large number of unknown quantities, a general scaling relation of the following form can be derived:

$\mathrm{Nu}=\alpha\langle L\rangle^{\gamma} \mathrm{Ra}_{\mathrm{har}}^{\beta}$,

where $\langle L\rangle$ is the average lid thickness. A least square fit to the above relation of all the available data, including diffusion creep models (Section 4.1), composite rheology models (Section 4.3), the dislocation creep model (Section 4.2) and Newtonian models with 
Table 2. Coefficients of the Nusselt-Rayleigh relationship $\mathrm{Nu}=\alpha \mathrm{Ra}^{\beta}$ for diffusion creep simulations obtained using different averages of the viscosity as discussed in Section 2.5. The coefficients are reported for the three regimes described in the text.

\begin{tabular}{lccc}
\hline & Method & $\alpha$ & $\beta$ \\
\hline Regime 1 & Arithmetic & 0.009 & 0.401 \\
& Geometric & 0.017 & 0.333 \\
& $\dot{\epsilon}^{1}$ & 0.012 & 0.369 \\
& $\dot{\epsilon}^{2}$ & 0.019 & 0.331 \\
& Harmonic & 0.036 & 0.266 \\
& Ra $_{\mathrm{r}}$ & 0.004 & 0.416 \\
\hline Regime 2 & Arithmetic & 0.083 & 0.250 \\
& Geometric & 0.051 & 0.258 \\
& $\dot{\epsilon}^{1}$ & 0.084 & 0.235 \\
& $\dot{\epsilon}^{2}$ & 0.081 & 0.219 \\
& Harmonic & 0.041 & 0.251 \\
& Ra $_{\mathrm{r}}$ & 0.052 & 0.246 \\
\hline Arithmetic & 0.042 & 0.300 \\
& Geometric & 0.049 & 0.268 \\
$\dot{\epsilon}^{1}$ & 0.056 & 0.264 \\
& $\dot{\epsilon}^{2}$ & 0.084 & 0.223 \\
& Harmonic & 0.054 & 0.242 \\
& Ra $a_{\mathrm{r}}$ & 0.076 & 0.230 \\
\hline
\end{tabular}

various activation enthalpies (Section 4.4) yields

$\alpha=0.370$

$\beta=0.071$

$\gamma=-0.666$,

using the harmonic average of the viscosity to compute the effective Rayleigh number.

In Fig. 11 we compare the Nusselt numbers obtained from the numerical simulations with the predictions of eq. (36). With the above values of $\alpha, \beta$ and $\gamma$, eq. (36) predicts the Nusselt number with a maximal deviation of 6 per cent. Only 9 of the 115 simulations that we ran show an error of more than 0.1 (the maximum is 0.25 ), all others deviate from the calculated value only in the second (or higher) decimal place. This scaling is therefore at least as good as the ones discussed above based on the generalized FrankKamenetskii parameter. The need to determine the mean lid thickness in eq. (36) poses a similar restriction as the determination of the Frank-Kamentskii parameter (which requires knowing $T_{i}$ and $P_{i}$ ), but limits the number of parameters that have to be known. Eq. (36) shows that for stagnant lid convection the heat flux is independent of the specific deformation mechanism or the shape of the stagnant lid. Only the thickness of the stagnant lid and the viscosity below it matter. Neither the activation energy, nor the activation volume, nor the stress exponent are required.

In Fig. 12 we show a contour plot of the Nusselt number as a function of the mean lid thickness and the effective Rayleigh number $\left(R a_{\text {har }}\right)$ calculated with eq. (36). The thicker the lid is, the less the Nusselt number is affected by the Rayleigh number. This is a consequence of the insulating nature of the stagnant lid. The scaling relation is only valid when a stagnant lid is developed and cannot be adopted for models without a stagnant lid. Each of the shaded points corresponds to one simulation that we used to find the scaling parameters. Thus, they represent the valid parameter space for the scaling.

The above scaling (eq. (36)) requires the thickness of the stagnant lid to be specified. In order to apply this relation in parametrized models, an equation for the time evolution of the lid thickness could be implemented that is based on the heat flux at the base of the lid (e.g. Spohn 1990). This would additionally require knowledge of the temperature contrast across the upper thermal boundary layer, which is again a function of the specific rheology chosen and cannot be easily determined. For a Newtonian rheology with viscosity dependent only on temperature, a scaling of the form:

$T_{\text {lid }}=T_{\mathrm{i}}-c \frac{R T_{\mathrm{i}}^{2}}{E}$,

is usually applied (e.g. Grasset \& Parmentier 1998), were the second term on the right-hand side is the inverse of the Frank-Kamentskii parameter and $c$ a constant. Our models runs are based on temperature, pressure and strain rate. Thus eq. (37) is not easily applicable with our rheology. Whether our scaling (eq. 36) holds also for a purely temperature-dependent viscosity would have to be investigated further with dedicated simulations.

\section{DISCUSSION AND CONCLUSIONS}

Despite the relative simplicity of the numerical model, our results contain a number of findings that can be used as a starting point for future studies on convection with complex rheologies in stagnantlid bodies. A composite rheology generally shows a higher heat transport efficiency for the same grain size compared to the pure diffusion creep models (Fig. 5a). Also, when using a composite rheology, the presence of dislocation creep has a strong influence on the thickness and shape of the stagnant lid. The lid tends to have a large slope when the rheology is based on diffusion creep while it is relatively flat as soon as dislocation creep is accounted for (Fig. 4). This is particularly important when considering melt production. A large slope is typically created by upwelling plumes, which easily induce partial melting near the base of the lid. In contrast, melt production is strongly reduced in the presence of a flat lid caused by dislocation creep. For a given grain size, composite rheology convection systematically leads to a lower melt production than pure diffusion creep (Fig. 3b).

As expected, diffusion creep dominates over dislocation creep when the grain size is small. The absolute value, however, strongly depends on the local temperature, pressure and strain rate. For our set of parameters relevant for a Mars-like planet, $d$ must be smaller than $\approx 2.5 \mathrm{~mm}$ to ensure that diffusion creep dominates over dislocation creep. By contrast, the latter is the dominant deformation mechanism for $d$ larger than $\approx 8 \mathrm{~mm}$ (Fig. 3a). Combining our findings with those of van den Berg et al. (1993, 1995), who suggested that dislocation creep becomes more important with increasing convective vigour, a grain size of $2.5 \mathrm{~mm}$ can be interpreted as the upper threshold to ensure that diffusion creep dominates the deformation over the whole domain. Increasing the temperature contrast across the mantle or including heat sources - that is increasing the convective vigour - will most likely lead to the increase of the dislocation creep fraction for comparable grain sizes.

By modelling the internal dissipation due to tides raised by Phobos, Nimmo \& Faul (2013) concluded that Mars' tidal quality factor and Love number $k_{2}$ can be best reproduced by a model of viscoelastic deformation with a grain size of $1 \mathrm{~cm}$ and a present-day mantle potential temperature of $1625 \pm 75 \mathrm{~K}$, in agreement with petrological estimates (Baratoux et al. 2011). Based on our results, the inferred grain size of $1 \mathrm{~cm}$ suggests that convection in the Martian mantle should be presently controlled entirely by dislocation creep (Fig. 3a). For the pure dislocation creep model (Fig. 2), we obtain in steady state a potential temperature of about $1600 \mathrm{~K}$ (not shown), in good agreement with the above estimate. Nevertheless, as discussed 

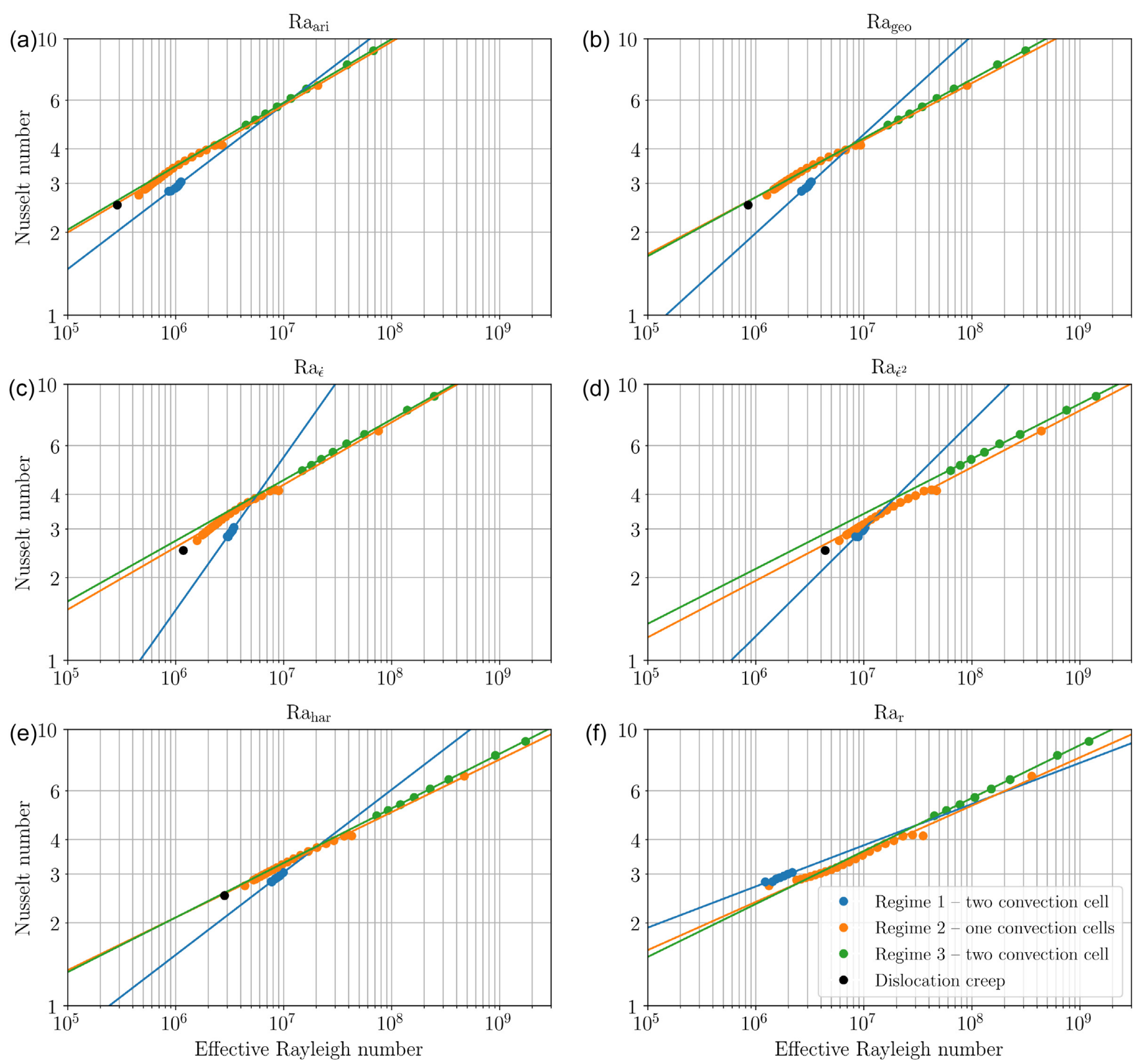

Figure 9. As in Fig. 8 but for simulations with composite rheology.

in Section 4.2, the thick stagnant lid associated with this regime prevents the formation of partial melt, a requirement used by Nimmo \& Faul (2013) to constrain their models. Yet, Nimmo \& Faul (2013) assumed a significantly smaller lid thickness $(125 \mathrm{~km}$ instead of about $400 \mathrm{~km}$ as obtained from our model), which explains why partial melt occurs in their case but not in our despite the similar potential temperatures.

At any rate, our models are too simple to be directly applicable to Mars. In particular, internal heating-whose neglect is actually appropriate when using a Cartesian geometry (O'Farrell et al. 2013)and the enrichment of heat producing elements in the crust (Plesa et al. 2018) would lead to different temperatures that could affect the range of grain sizes across which we observe the transition from diffusion-creep-dominated to dislocation-creep-dominated convection, as well as the lid thickness and hence the possibility to generate partial melt. Furthermore, other authors predicted different grain sizes for present-day Mars. Khan et al. (2018) suggested only

a lower bound on the grain size of about $1 \mathrm{~mm}$. Plesa et al. (2018) found that the tidal quality factor, $k_{2}$ Love number and various other constraints on the thermal evolution of Mars can be satisfied by models for which the grain size is between $12 \mathrm{~mm}$ and $4 \mathrm{~cm}$, thus indicating that dislocation creep is likely to play an important role for mantle convection in Mars, despite large uncertainties and limitations of our models.

Notably, in a recent study of the thermal evolution of Mars - but based on 1-D models of parametrized convection-Samuel et al. (2019) showed that a low effective activation energy indicative of dislocation creep is required to satisfy various observational constraints, particularly the stability of the orbit of Phobos. As discussed in Section 2.4, in studies of mantle dynamics and thermal evolution of stagnant-lid bodies, a linear diffusion creep rheology is often used in place of non-linear dislocation creep following the early suggestion of Christensen (1984) according to which the latter can be mimicked by an effective linear rheology with reduced activation 
Table 3. Coefficients of the Nusselt-Rayleigh relationship $\mathrm{Nu}=\alpha \mathrm{Ra}^{\beta}$ for composite rheology simulations obtained using different averages of the viscosity as discussed in Section 2.5. The coefficients are reported for the three regimes described in the text.

\begin{tabular}{lcccc}
\hline & & & & $R^{2}$ \\
& Method & $\alpha$ & $\beta$ & (per cent) \\
\hline Regime 1 & Arithmetic & 0.048 & 0.298 & 93.74 \\
& Geometric & 0.014 & 0.357 & 96.99 \\
& $\dot{\epsilon}^{1}$ & 0.001 & 0.553 & 97.17 \\
& $\dot{\epsilon}^{2}$ & 0.006 & 0.388 & 91.89 \\
& Harmonic & 0.025 & 0.299 & 99.19 \\
& $\mathrm{Ra}_{\mathrm{r}}$ & 0.345 & 0.149 & 95.9 \\
\hline Regime 2 & Arithmetic & 0.141 & 0.230 & 99.31 \\
& Geometric & 0.155 & 0.206 & 99.49 \\
& $\dot{\epsilon}^{1}$ & 0.114 & 0.226 & 98.99 \\
& $\dot{\epsilon}^{2}$ & 0.115 & 0.205 & 99.37 \\
& Harmonic & 0.150 & 0.190 & 99.68 \\
& Ra ${ }_{\mathrm{r}}$ & 0.214 & 0.174 & 98.05 \\
\hline Regime 3 & Arithmetic & 0.145 & 0.230 & 99.96 \\
& Geometric & 0.141 & 0.213 & 99.97 \\
& $\dot{\epsilon}^{1}$ & 0.131 & 0.220 & 99.94 \\
& $\dot{\epsilon}^{2}$ & 0.138 & 0.200 & 99.95 \\
& Harmonic & 0.136 & 0.197 & 99.98 \\
& Ra & 0.166 & 0.191 & 99.95 \\
\hline
\end{tabular}

(a)

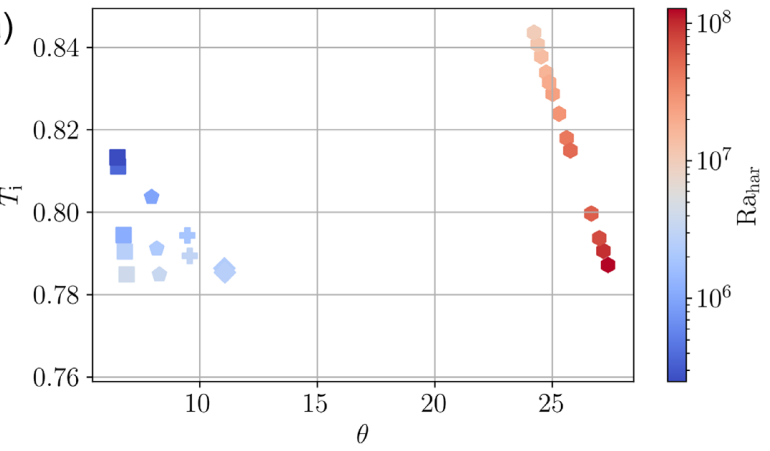

(b)

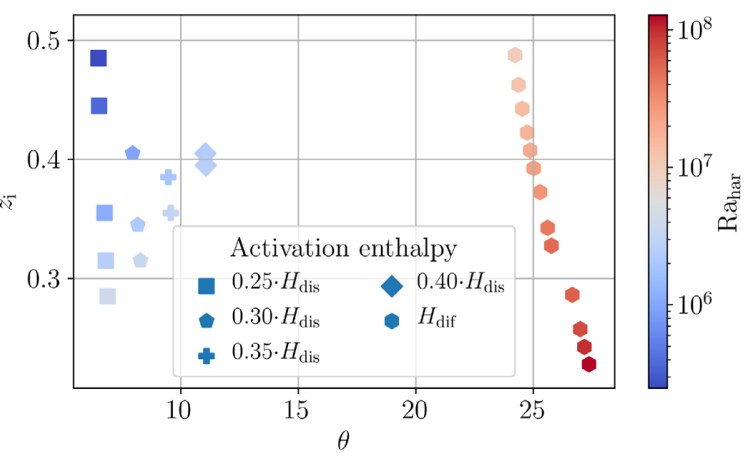

Figure 10. (a) Internal temperature $T_{\mathrm{i}}$ and (b) depth of the base of the upper thermal boundary layer $z_{\mathrm{i}}$ as a function of the generalized FrankKamenetskii parameter $\theta$ and effective Rayleigh number $\mathrm{Ra}_{\text {har }}$ based on the harmonic average. Only simulations with one convection cell and a lid with steep slope are plotted here. Symbols indicate different fractions of the activation energy of dislocation creep. Red symbols with high values of $\mathrm{Ra}_{\text {har }}$ and $\theta$ correspond to standard diffusion creep simulations, while blue symbols correspond to diffusion creep simulations with activation enthalpy of dislocation creep $H_{\mathrm{dis}}$, reduced according to the factors indicated in the legend.

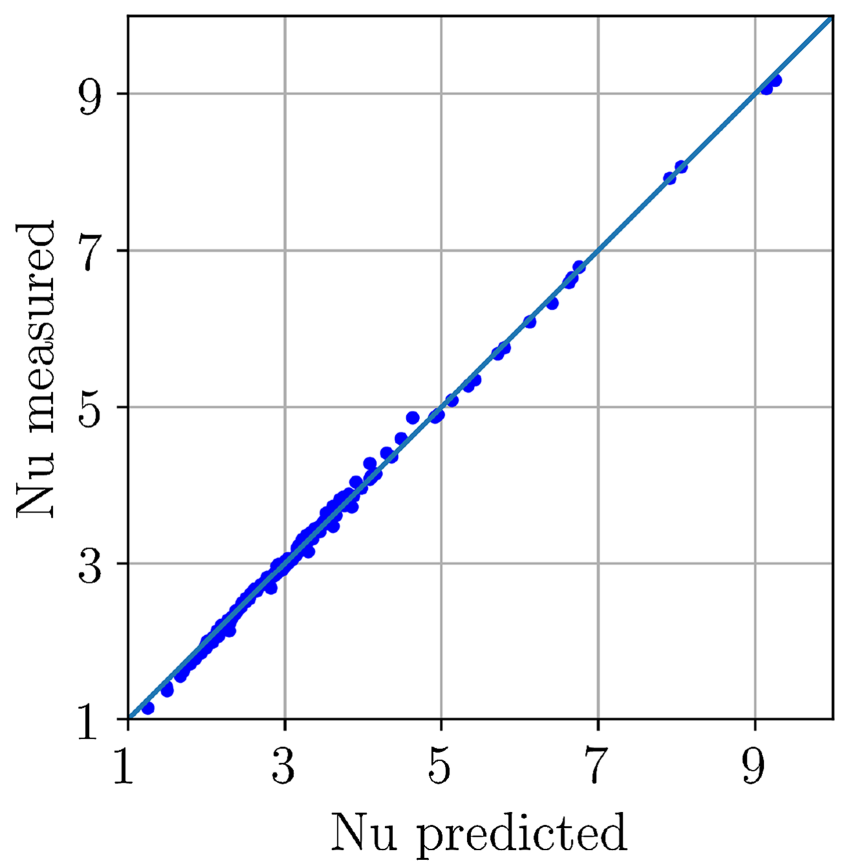

Figure 11. Measured Nusselt number as a function of the Nusselt number predicted by eq. (36) for all simulations.

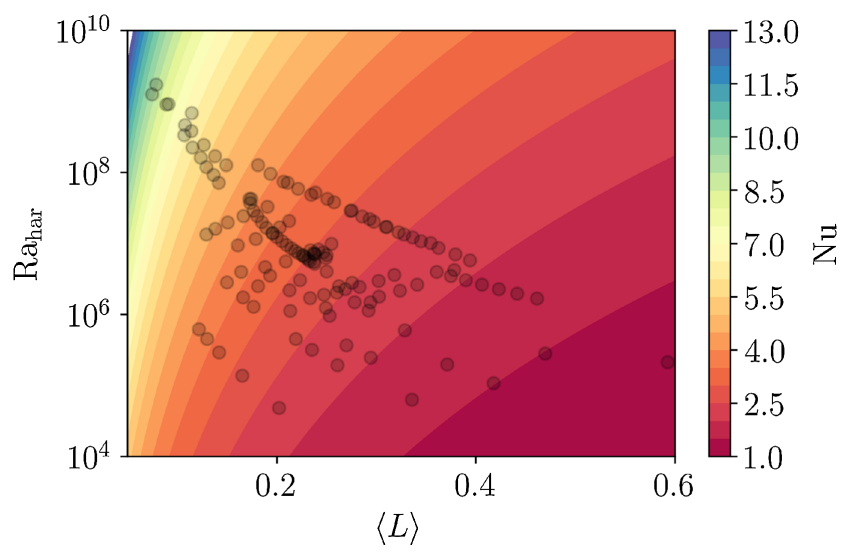

Figure 12. Nusselt number calculated with eq. (36) as a function of the effective Rayleigh number based on the harmonic average of the viscosity and the average lid thickness. Each of the shaded points corresponds to one simulation that we used to find the scaling parameters.

enthalpy. For the first time, we explored in detail this idea and the extent to which it is applicable. Mimicking dislocation creep by reducing the activation enthalpy as done by Christensen (1984) - but in the absence of a stagnant lid-has a limited validity. The Nusselt number, mean lid thickness, mean temperature and rms velocity can be reproduced but not simultaneously and for the same reduction factor (Fig. 7). Mimicking the flow pattern and lid shape is not possible. Since the slope of the lid is mostly influenced by the temperature dependence of the viscosity, the activation energy and volume would likely need to be modified independently to obtain a good match. The pressure dependence can be used to increase or decrease the lid thickness, while the temperature dependence to adjust the velocity and flow pattern. If internal heating were also taken into account, the temperature contrast between up- and downwellings would possibly be lower and thus the lid slope less steep. In this case, a simple reduction of the activation enthalpy could be 
sufficient to reproduce the flow pattern obtained by using dislocation creep. This could be investigated in future studies. However, the large number of special cases to be considered shows that such an approach is not advisable for realistic thermal evolution models, as most of these flow properties are time-dependent.

In pure diffusion creep models with constant activation energy and volume we found that a single combination of pre-factor $\alpha$ and exponent $\beta$ can not fulfill the relation $\mathrm{Nu}=\alpha \mathrm{Ra}^{\beta}$ for all Rayleigh numbers. $\alpha$ and $\beta$ rather depend on the characteristic flow pattern. Although this behaviour is the same for the composite rheology, considering Newtonian diffusion creep and non-Newtonian dislocation creep, the scaling parameters are different. Even though the fraction of dislocation creep increases with decreasing Rayleigh number, the scaling parameters remain constant in each of the three regimes. This suggests that the impact of the stress exponent $(n)$ on $\beta$ is negligible.

The implementation of a characteristic value for the temperature and pressure dependence of the viscosity (the Frank-Kamenetskii parameter $\theta$ ) into the scaling relation requires knowledge of the depth of the base of the upper thermal boundary layer, the shape of the lid, the internal temperature and the deformation mechanism acting (e.g. Fowler 1985; Reese et al. 1998). In the framework of our parameter study on grain size, not all of these parameters could be systematically investigated. Only for the Newtonian case, with a steep slope we were able to show that the dependence on $\theta$ is weaker than predicted, which is likely due to the pressure dependence of our rheological model. However, the characteristic values needed for the scaling law cannot be known beforehand. We thus scaled the Nusselt number only with the mean lid thickness and effective Rayleigh number based on the harmonic average of the viscosity and found $\mathrm{Nu}=0.37\langle L\rangle^{-0.666} \mathrm{Ra}_{\text {har }}^{0.071}$, which fits well all our model runs. The consequence of this scaling law is that the power dependence of the Rayleigh number does not depend on the chosen stress exponent. However, the lid thickness depends on the chosen rheology. Whether the lid thickness is determined based on the stress exponent, grain size or activation enthalpy plays a minor role. The shape of the lid does not matter either. Regardless of the fitting function used, the effective Rayleigh number based on the harmonic average of the viscosity, which corresponds to the viscosity value near the base of the stagnant lid, provides the most accurate estimate of the Nusselt number.

Overall, simulations based on pure diffusion creep and composite rheology differ considerably in terms of heat flux, lid shape, internal temperature and melt production. Together with the (limited) evidence for relatively large grain sizes at present-day, they suggest that models of the thermal evolution of Mars should be revaluated in light of the effects of a composite rheology combining diffusion and dislocation creep.

\section{ACKNOWLEDGEMENTS}

We thank two anonymous reviewers and the editor Gael Choblet for their constructive comments that helped to significantly improve a previous version of the manuscript. F. Schulz and N. Tosi acknowledge support from the DFG through the priority program SPP-1833 'Building a habitable Earth' (project no. TO 704/2-1). N. Tosi was also supported by the Helmholtz Association (project VH-NG-1017). A.-C. Plesa would like to gratefully acknowledge the financial support and endorsement from the DLR Management Board Young Research Group Leader Program and the Executive Board Member for Space Research and Technology. Computational time was provided by the HLRN (project bep00063), which is gratefully acknowledged.

\section{REFER E N CES}

Alisic, L., Gurnis, M., Stadler, G., Burstedde, C., Wilcox, L.C. \& Ghattas, O., 2010. Slab stress and strain rate as constraints on global mantle flow, Geophys. Res. Lett., 37(22), doi:10.1029/2010GL045312.

Austin, N.J. \& Evans, B., 2007. Paleowattmeters: a scaling relation for dynamically recrystallized grain size, Geology, 35, 343.

Baratoux, D., Toplis, M.J., Monnereau, M. \& Gasnault, O., 2011. Thermal history of mars inferred from orbital geochemistry of volcanic provinces, Nature, 472(7343), 338.

Billen, M.I. \& Hirth, G., 2007. Rheologic controls on slab dynamics, Geochem. Geophys. Geosyst., 8(8), doi:10.1029/2007GC001597.

Blackman, D.K. \& Kendall, J.-M., 2002. Seismic anisotropy in the upper mantle 2. Predictions for current plate boundary flow models, Geochem. Geophys. Geosyst., 3(9), doi:10.1029/2001GC000247.

Christensen, U., 1984. Convection with pressure- and temperaturedependent non-Newtonian rheology, Geophys. J., 77, 343-384.

Citron, R.I., Manga, M. \& Tan, E., 2018. A hybrid origin of the Martian crustal dichotomy: degree-1 convection antipodal to a giant impact, Earth planet. Sci. Lett., 491, 58-66.

Ćížková, H., van Hunen, J. \& van den Berg, A., 2007. Stress distribution within subducting slabs and their deformation in the transition zone, Phys. Earth. planet. Inter., 161(3-4), 202-214.

Dannberg, J., Eilon, Z., Faul, U., Gassmöller, R., Moulik, P. \& Myhill, R., 2017. The importance of grain size to mantle dynamics and seismological observations, Geochem. Geophys. Geosyst., 18, 3034-3061.

Elkins-Tanton, L.T., Zaranek, S.E., Parmentier, E. \& Hess, P., 2005. Early magnetic field and magmatic activity on Mars from magma ocean cumulate overturn, Earth planet. Sci. Lett., 236(1-2), 1-12.

Evans, B., Renner, J. \& Hirth, G., 2001. A few remarks on the kinetics of static grain growth in rocks, Int. J. Earth Sci., 90, 88-103.

Foley, B.J. \& Bercovici, D., 2014. Scaling laws for convection with temperature-dependent viscosity and grain-damage, Geophys. J. Int, 199(1), 580-603.

Fowler, A., 1985. Fast thermoviscous convection, Stud. Appl. Math., 72(3), 189-219.

Grasset, O. \& Parmentier, E., 1998. Thermal convection in a volumetrically heated, infinite Prandtl number fluid with strongly temperature-dependent viscosity: implications for planetary evolution, J. geophys. Res., 103, $18171-18181$.

Grigné, C., Labrosse, S. \& Tackley, P., 2007. Convection under a lid of finite conductivity in wide aspect ratio models: effect of continents on the wavelength of mantle flow, J. geophys. Res.: Solid Earth, 112(B8), doi:10.1029/2006JB004297.

Hirth, G. \& Kohlstedt, D., 2003. Rheology of the upper mantle and the mantle wedge: a view from the experimentalists, Wash. DC Am. Geophys. Un. Geophys. Monogr. Ser., 138, 83-105.

Hüttig, C., Tosi, N. \& Moore, W.B., 2013. An improved formulation of the incompressible Navier-Stokes equations with variable viscosity, Phys. Earth planet. Inter, 220, 11-18.

Kaminski, E., Ribe, N.M. \& Browaeys, J.T., 2004. D-Rex, a program for calculation of seismic anisotropy due to crystal lattice preferred orientation in the convective upper mantle, Geophys. J. Int., 158(2), 744-752.

Karato, S., 1998. Seismic anisotropy in the deep mantle, boundary layers and the geometry of mantle convection, Pure appl. Geophys., 151(2-4), 565 .

Karato, S.-I., 2012. Deformation of Earth Materials: An Introduction to the Rheology of Solid Earth, Cambridge University Press.

Karato, S.-I. \& Jung, H., 2003. Effects of pressure on high-temperature dislocation creep in olivine, Phil. Mag., 83(3), 401-414.

Karato, S.-I. \& Wu, P., 1993. Rheology of the upper mantle: a synthesis, Science, 260(5109), 771-778.

Karato,S.-I., Zhang, S. \& Wenk, H.-R., 1995. Superplasticity in Earth's lower mantle: evidence from seismic anisotropy and rock physics, Science, 270(5235), 458-461. 
Karato,S.-I., Riedel, M.R. \& Yuen, D.A., 2001. Rheological structure and deformation of subducted slabs in the mantle transition zone: implications for mantle circulation and deep earthquakes, Phys. Earth planet. Inter., 127(1-4), 83-108.

Katz, R.F., Spiegelman, M. \& Langmuir, C.H., 2003. A new parameterization of hydrous mantle melting, Geochem. Geophys. Geosyst., 4(9), 1073.

Kawada, Y. \& Honda, S., 1999. Parametrization of heat transport by nonNewtonian convection, Geophys. J. Int, 137, 441-448.

Khan, A., Liebske, C., Rozel, A., Rivoldini, A., Nimmo, F., Connolly, J.A.D., Plesa, A.C. \& Giardini, D., 2018. A geophysical perspective on the bulk composition of Mars, J. geophys. Res.: Planets, 123(2), 575-611.

King, S.D., Lee, C., van Keken, P.E., Leng, W., Zhong, S., Tan, E., Tosi, N. \& Kameyama, M.C., 2010. A community benchmark for 2-D Cartesian compressible convection in the Earth's mantle, Geophys. J. Int, 180, 7387.

Laneuville, M., Wieczorek, M., Breuer, D. \& Tosi, N., 2013. Asymmetric thermal evolution of the Moon, J. geophys. Res.: Planets, 118(7), 14351452.

McNamara, A.K., Karato, S.-I. \& van Keken, P.E., 2001. Localization of dislocation creep in the lower mantle: implications for the origin of seismic anisotropy, Earth planet. Sci. Lett., 191, 85-99.

Moresi, L.-N. \& Solomatov, V.S., 1995. Numerical investigation of 2d convection with extremely large viscosity variations, Phys. Fluids, 7(9), 2154-2162.

Morris, S. \& Canright, D., 1984. A boundary-layer analysis of benard convection in a fluid of strongly temperature-dependent viscosity, Phys. Earth planet. Inter., 36, 355-373.

Nakagawa, T. \& Tackley, P.J., 2015. Influence of plate tectonic mode on the coupled thermochemical evolution of Earth's mantle and core, Geochem. Geophys. Geosyst., 16(10), 3400-3413.

Nimmo, F. \& Faul, U.H., 2013. Dissipation at tidal and seismic frequencies in a melt-free, anhydrous Mars, J. geophys. Res.: Planets, 118, 2558-2569.

Noack, L. \& Breuer, D., 2013. First- and second-order Frank-Kamenetskii approximation applied to temperature-, pressure- and stress-dependent rheology, Geophys. J. Int, 195, 27-46.

O'Farrell, K.A., Lowman, J.P. \& Bunge, H.-P., 2013. Comparison of spherical-shell and plane-layer mantle convection thermal structure in viscously stratified models with mixed-mode heating: implications for the incorporation of temperature-dependent parameters, Geophys. J. Int., 192(2), 456-472.

Padovan, S., Tosi, N., Plesa, A.-C. \& Ruedas, T., 2017. Impact-induced changes in source depth and volume of magmatism on Mercury and their observational signatures, Nature Comm., 8, doi:10.1038/s41467017-01692.

Parmentier, E.M., Turcotte, D.L. \& Torrance, K.E., 1976. Studies of finite amplitude non-Newtonian thermal convection with application to convection in the earth's mantle, J. geophys. Res., 81, 1839-1846.

Plesa, A.C. \& Breuer, D., 2014. Partial melting in one-plate planets: implications for thermo-chemical and atmospheric evolution, Planet. Space Sci., 98, 50-65.

Plesa, A.-C., Tosi, N., Grott, M. \& Breuer, D., 2015. Thermal evolution and Urey ratio of Mars, J. geophys. Res.: Planets, 120(5), 995-1010.

Plesa, A.-C. et al., 2018. The thermal state and interior structure of Mars, Geophys. Res. Lett., 45, 12.

Redmond, H.L. \& King, S.D., 2007. Does mantle convection currently exist on Mercury? Phys. Earth planet. Inter., 164(3-4), 221-231.

Reese, C., Solomatov, V. \& Moresi, L.-N., 1998. Heat transport efficiency for stagnant lid convection with dislocation viscosity: application to Mars and Venus, J. geophys. Res.: Planets, 103(E6), 13643-13657.

Reese, C.C., Solomatov, V.S. \& Moresi, L.-N., 1999. Non-Newtonian stagnant lid convection and magmatic resurfacing on Venus, Icarus, 139, $67-80$.

Ricard, Y. \& Bercovici, D., 2009. A continuum theory of grain size evolution and damage, J. geophys. Res.: Solid Earth, 114, B01204.

Roberts, G.O., 1979. Fast viscous Bénard convection, Geophys. Astrophys. Fluid Dyn., 12(1), 235-272.
Roberts, J.H. \& Barnouin, O.S., 2012. The effect of the Caloris impact on the mantle dynamics and volcanism of Mercury, J. geophys. Res.: Planets, 117(E2), E02007.

Rolf, T., Zhu, M.-H., Wünnemann, K. \& Werner, S.C., 2016. The role of impact bombardment history in lunar evolution, Icarus, 286, 138-152.

Rozel, A., 2012. Impact of grain size on the convection of terrestrial planets, Geochem. Geophys. Geosyst., 13, Q10020.

Rozel, A., Ricard, Y. \& Bercovici, D., 2011. A thermodynamically selfconsistent damage equation for grain size evolution during dynamic recrystallization, Geophys. J. Int., 184, 719-728.

Ruedas, T. \& Breuer, D., 2017. On the relative importance of thermal and chemical buoyancy in regular and impact-induced melting in a Mars-like planet, J. geophys. Res.: Planets, 122(7), 1554-1579.

Samuel, H. \& Tosi, N., 2012. The influence of post-perovskite strength on the earth's mantle thermal and chemical evolution, Earth planet. Sci. Lett., 323, 50-59.

Samuel, H., Aleksandrov, V. \& Deo, B., 2011. The effect of continents on mantle convective stirring, Geophys. Res. Lett., 38(4), doi:10.1029/2010GL04605

Samuel, H., Lognonné, P., Panning, M. \& Lainey, V., 2019. The rheology and thermal history of Mars revealed by the orbital evolution of Phobos, Nature, 569(7757), 523-527.

Scheinberg, A., Elkins-Tanton, L. \& Zhong, S., 2014. Timescale and morphology of Martian mantle overturn immediately following magma ocean solidification, J. geophys. Res., 119(3), 454-467.

Scheinberg, A.L., Soderlund, K.M. \& Elkins-Tanton, L.T., 2018. A basal magma ocean dynamo to explain the early lunar magnetic field, Earth planet. Sci. Lett., 492, 144-151.

Sekhar, P. \& King, S.D., 2014. 3D spherical models of Martian mantle convection constrained by melting history, Earth planet. Sci. Lett., $\mathbf{3 8 8 ,}$ 27-37.

Solomatov, V.S. \& Moresi, L.-N., 2000. Scaling of time-dependent stagnant lid convection: application to small-scale convection on earth and other terrestrial planets, J. geophys. Res.: Solid Earth, 105, 21 795-21 818.

Spohn, T., 1990. Mantle differentiation and thermal evolution of Mars, Mercury, and Venus, Icarus, 90, 1222-236.

Šrámek, O. \& Zhong, S., 2010. Long-wavelength stagnant lid convection with hemispheric variation in lithospheric thickness: link between Martian crustal dichotomy and Tharsis? J. geophys. Res.: Planets, 115(E9), doi:10.1029/2010JE003597.

Stein, C. \& Hansen, U., 2013. Arrhenius rheology versus FrankKamenetskii rheology-Implications for mantle dynamics, Geochem. Geophys. Geosyst., 14(8), 2757-2770.

Tosi, N., Grott, M., Plesa, A.-C. \& Breuer, D., 2013. Thermochemical evolution of Mercury's interior, J. geophys. Res.: Planets, 118(12), 2474-2487.

Tosi, N., Maierová, P. \& Yuen, D.A., 2015. Influence of variable thermal expansivity and conductivity on deep subduction, in Subduction Dynamics: From Mantle Flow to Mega Disasters, pp. 115-133, eds Morra, G., Yuen, D.A., King, S.D. \& Stein, S., American Geophysical Union.

Turcotte, D. \& Oxburgh, E., 1967. Finite amplitude convective cells and continental drift, J. Fluid Mech., 28(1), 29-42.

van den Berg, A.P., van Keken, P.E. \& Yuen, D.A., 1993. The effects of a composite non-Newtonian and Newtonian rheology on mantle convection., Geophys. J. Int, 115, 62-78.

van den Berg, A.P., Yuen, D.A. \& van Keken, P.E., 1995. Rheological transition in mantle convection with a composite temperature-dependent, non-Newtonian and Newtonian rheology, Earth planet. Sci. Lett., 129, 249-260.

Wong, T. \& Solomatov, V.S., 2015. Towards scaling laws for subduction initiation on terrestrial planets: constraints from two-dimensional steadystate convection simulations, Prog. Earth planet. Sci., 2, 18.

Zhang, N., Parmentier, E.M. \& Liang, Y., 2013. A 3-D numerical study of the thermal evolution of the Moon after cumulate mantle overturn: the importance of rheology and core solidification, J. geophys. Res.: Planets, 118, 1789-1804.

Zhang, S. \& O'Neill, C., 2016. The early geodynamic evolution of Mars-type planets, Icarus, 265, 187-208. 